\title{
Genus generators and the positivity of the signature
}

\author{
A Stoimenow
}

\begin{abstract}
It is a conjecture that the signature of a positive link is bounded below by an increasing function of its negated Euler characteristic. In relation to this conjecture, we apply the generator description for canonical genus to show that the boundedness of the genera of positive knots with given signature can be algorithmically partially decided. We relate this to the result that the set of knots of canonical genus $\geq n$ is dominated by a finite subset of itself in the sense of Taniyama's partial order.
\end{abstract}

57M25; $57 \mathrm{~N} 70$

\section{Introduction and motivation}

\subsection{The signature growth conjecture}

Most of the introductory exposition is similar, or identical to [33]. A positive link (see, eg, Cromwell [8], Ozawa [26], Yokota [51]) is a link which can be represented by a positive diagram. Such links occur in several contexts, eg, the theory of dynamical systems (Franks and Williams [12; 49]), singularity theory (A'Campo [1], Boileau and Weber [3], Rudolph [32]), and (in some vague and yet-to-be understood way) in 4-dimensional QFTs (Kreimer [18]). They contain the class of positive braid links (see van Buskirk [47], Cromwell [9]), the closure links of positive braids ${ }^{1}$. Another important subclass of the class of positive links are the alternating ones among them, the special alternating links (see Cromwell [8], Nakamura [25]). Such links have been studied largely by Murasugi [23; 24].

Knot-theoretically, one is interested how positivity can be detected by the examination of link invariants. One of the most classical such invariants is the signature $\sigma$. It was studied initially by Murasugi [24], and is defined in terms of Seifert matrices. It thus has a natural upper bound. For a general link $L$, let $\chi(L)$ be the maximal Euler characteristic of a spanning Seifert surface, and $n(L)$ the number of components. Then $\sigma(L) \leq n(L)-\chi(L)$, and if $L$ has no split components bounding disconnected Seifert

\footnotetext{
${ }^{1}$ Beware that some authors, for example van Buskirk [47], confusingly call 'positive links' what we call 'braid positive links' here. Other authors call our braid positive links 'positive braids', abusing the distinction between braids and their closures.
} 
surfaces (as for example for positive or alternating links), this estimate modifies to $\sigma(L) \leq n^{\prime}(L)-\chi(L)$, with $n^{\prime}(L)$ being the number of split components of $L$.

The positivity of the signature on positive links (or subclasses thereof) has been a theme occurring throughout the literature over a long period. The first result falling into this category was established already by Murasugi in his initial study [24]. He showed that the upper bound in terms of $\chi$ is exact for a special alternating link [24]. This is found not to be true for general positive, or positive braid links by means of simple examples.

Motivated by their study in dynamical systems, in [30], Lee Rudolph showed that (non-trivial) braid positive links have (at least) strictly positive ${ }^{2}$ signature $\sigma$. This result was subsequently extended to positive links by Cochran and Gompf [6, corollary 3.4]. A different proof, proposed by Traczyk [46], has unfortunately a gap and breaks down at least partly. (It still applies for positive braid knots, the special case settled previously by Rudolph.) Przytycki observed the result (also for almost positive knots) to be a consequence of Taniyama's work [45], but a draft with an account on the subject was not finished. A related proof was written down in [42].

It is suggestive to ask how much more the signature of positive links can grow. One should believe in an increase of $\sigma$, in the range between the maximal value in Murasugi's result and the mere positivity property. Some evidence suggests the following conjecture, mentioned first explicitly in [37]. (See Section 1.3 for some discussion of this evidence.)

Conjecture 1.1 (Signature Growth conjecture)

$$
\liminf _{n \rightarrow \infty} \min \left\{\sigma(L): L \text { positive link, } \chi^{\prime}(L)=n\right\}=\infty,
$$

where for a link $L$ we set $\chi^{\prime}(L):=n^{\prime}(L)-\chi(L)$.

Alternatively speaking, one asks whether

$$
\Sigma_{\sigma}=\left\{\chi^{\prime}(L): L \text { positive link, } \sigma(L)=\sigma\right\}
$$

is finite for every $\sigma$.

This conjecture, although suggestive, is by no means obvious, or easily approachable. Although $\sigma$ is easily calculated for any specific link, it has turned out difficult to make general statements about it on large link classes. This situation is a bit opposite to $\chi$, for which much more general formulas are available, but whose calculation for specific links (falling outside the "nice" classes) may be very complicated.

\footnotetext{
${ }^{2}$ There is often confusion about the choice of sign in the definition of $\sigma$. Here (following [30], rather than [46] or [6]), we use the more natural seeming convention that positive links have positive, and not negative $\sigma$.
} 


\subsection{Concordance and Bennequin's inequality}

A famous conjecture of Milnor states that for torus knots (or more generally knots of singularities) the smooth 4-ball genus is equal to the genus (or unknotting number, see Boileau and Weber [3]). This conjecture was settled later by gauge-theoretic work of Rudolph [31; 32] and Kronheimer-Mrowka (see [19]), which implied the (smooth) $4-$ genus version of the Bennequin inequality [2, theorem 3]. This inequality gives a lower bound for the genus in terms of a braid representation of a knot or link, and was used in his discovery of non-standard contact structures on $\mathbb{R}^{3}$. For a positive knot/link, the inequality estimates sharply the genus, and hence its newer version the 4-genus. So one obtains explicit formulas for these invariants, and for braid positive knots/links from [3] also for the unknotting/unlinking number. The (rather obvious) discussion can be found for example in $[17 ; 41]$.

One of Murasugi's original results about $\sigma$ is that it is a knot concordance invariant and estimates (from below) the 4-genus of a knot. The signature of torus knots (and links) was found by Gordon, Litherland and Murasugi [14] and Hirzebruch [16], and fails to provide the sharp estimate desired for Milnor's conjecture. Many more examples illustrate that the signature does not conform to the lower bound in Bennequin's inequality. Such examples led to the question, encountered already in Bennequin's original work, how to modify his inequality to be applicable also to $\sigma$. A solution was proposed in [33].

Recently, new signature-type concordance invariants, giving lower bounds for the 4-genus, were developed from Floer homology [27] and Khovanov's homology [28] theory. Positive knots are again intrinsically linked to these invariants, in that this time the 4-genus estimate is exact for such knots. (In particular, Rasmussen's approach gives a new, combinatorial, proof of Milnor's conjecture.)

One important difference between $\sigma$ and its successors is that only former is an invariant in the topological category, while latter apply only in the smooth category. This difference must be emphasized in view of the growing division in methods to study both types of concordance, where the Floer-Khovanov homological invariants on the smooth side contrast Levine's approach using the algebraic knot concordance group [20] and its recent non-abelian modifications due to Cochran-Orr-Teichner (see [5]) on the topological side.

Still serious problems to understand topological concordance, and its difference to smooth concordance, remain. Our knowledge about this question seems to center around Freedman's result that all knots with trivial Alexander polynomial are topologically slice. Some are known to be not smoothly slice. The first examples were given by 
Andrew Casson in the 1980s, using work of Donaldson. It was not before these deep results that one understood topological and smooth concordance are not the same. More such examples followed from later work of Fintushel-Stern [11] and Rudolph (see [30; 31]), but still they remain scarce even by now; all have trivial Alexander polynomial and rely on Freedman's criterion. (Recently Friedl and Teichner proposed some new candidates, with the Alexander polynomial of $6_{1}$, but their smooth non-sliceness status remains unclear so far. Their simplest good candidate for a possibly not smoothly slice knot has a diagram with 93 crossings.) With this state-of-the-art, one realizes to have obtained only limited understanding of topological concordance, and so the study of $\sigma$, a basic topological concordance invariant, gains new motivation.

\subsection{Statement of main result}

In a previous paper [33], we settled the case of positive braid links in Conjecture 1.1.

Theorem 1.2 [33]

$$
\liminf _{n \rightarrow \infty} \min \left\{\sigma(L): L \text { braid positive link, } \chi^{\prime}(L)=n\right\}=\infty
$$

This paper is a sequel to [33]; its motivation and problem setting is almost equivalent; the separation was made on the one hand for length reasons, on the other hand because the methods applied differ somewhat. In [33], we used an extension of Bennequin's inequality to $\sigma$. Here we will use another important ingredient, the generator description for canonical genus.

To state our main result it is helpful to understand the Growth conjecture in terms of the finiteness of the sets (1). For simplicity, consider below only knots and replace $\chi^{\prime}=1-\chi$ by the genus $g$ in (1). We start with a few remarks on known results about $\Sigma_{\sigma}$, providing hints to the Growth conjecture. First, the positivity result for $\sigma$ means $\Sigma_{0}=\{0\}$. Then, slightly implicitly in [45], and later independently and explicitly in [35], the result $\sigma>0$ was extended by showing that the only positive knots of $\sigma=2$ are those of genus one. Thus $\Sigma_{2}=\{1\}$. For $\sigma=4$ the situation is not that simple. Beside genus 2, there are some positive knots of genus 3 with $\sigma=4$, and one knot of genus $4,14_{45657}$ of [37]. Calculations of [37] suggest that $14_{45657}$ is in fact the only positive knot of genus 4 with $\sigma=4$, and for genus $g \geq 5$ indeed there seems no further such knot, that is, apparently $\Sigma_{4}=\{2,3,4\}$. Although this is still difficult to check, we will resolve the problem at least theoretically.

Our aim will be to show how one can prove, at least in theory, that any initial number of the sets $\Sigma_{\sigma}$ is finite, provided this is true. (Note that if $\Sigma_{\sigma}$ is infinite, then so is $\Sigma_{\sigma^{\prime}}$ for any $\sigma^{\prime}>\sigma$.) Namely, we show that there exists an algorithmically determinable 
collection of knots, such that if $\Sigma_{\sigma}$ is finite, only finitely many of the determined knots need to be checked to establish this finiteness.

Theorem 1.3 (Main result) For all $n>1$ there is a set $C_{n}$ of positive knots with two properties:

(1) $C_{n}$ is finite and algorithmically constructible.

(2) For all $\sigma \in 2 \mathbb{N}$ we have:

$\exists$ positive knot $K$ of genus $g \geq n$ with $\sigma(K) \leq \sigma \Longleftrightarrow \exists K \in C_{n}$ with $\sigma(K) \leq \sigma$.

To verify, using this theorem, that $\Sigma_{\sigma}$ is finite, one uses induction on $\sigma$. We know $\Sigma_{\sigma^{\prime}}$ for $\sigma^{\prime}<\sigma$ by induction. Then one examines

$$
C=C_{\max } \bigcup_{\sigma^{\prime} \leq \sigma} \Sigma_{\sigma^{\prime}}+1
$$

If some $K \in C$ is found with $g(K) \notin \Sigma_{\sigma}$ and $\sigma(K)=\sigma$, include $g(K)$ into $\Sigma_{\sigma}$, and repeat the search for such $K$ (with the new set $C$ updated according to (2)). If $\Sigma_{\sigma}$ is finite, after some iterations no $K$ will be found.

We remark that the same type of statement is true for links of any arbitrary fixed number of components. We do not prove it, however, in this more general form, since this generalization does not involve significantly new arguments, and would add only considerable technicality to the proof. We do elaborate on the knot case, though, giving different arguments that contribute to making $C_{n}$ as small as we can. We note that in the above theorem, one can replace $\sigma$ by any of the generalized (Tristram-Levine) signatures.

In the proof of Theorem 1.3, Hirasawa's algorithm [15], that lay in the center of the signature Bennequin inequality in [33], finds its application again, this time in combination with the generator theory for diagrams of given canonical genus, initiated in [38], and then developed further in [43; 44]. Namely, we use our result of [36] concerning the maximal crossing number of a generating diagram of a given genus. It improves a previous result of [44] in this regard, and relies heavily on Hirasawa's algorithm. The (original) Bennequin inequality also enters into the proof.

We mentioned the relation (noted by Przytycki) between the positivity of $\sigma$ and Taniyama's work [45]. We use this relation to bring his partial order into the context of our arguments. It follows from our proof of Theorem 1.3 that Taniyama's statements about the dominance of the trefoil and $5_{1}$ are the first two instances of a infinite series of such results, namely, that the set of knots of canonical genus $\geq n$ is dominated by a 
finite subset of itself (Theorem 5.6). A similar outcome, for positive knots, addresses the partial order of Cochran and Gompf [6] (Theorem 5.9).

Further evidence for the Growth conjecture is given by the following result on the average value of $\sigma$ for given genus.

Theorem 1.4 [36] Let

$$
P_{g, n}:=\{K \text { positive knot, } g(K)=g, c(K) \leq n\},
$$

where $c(K)$ denotes the crossing number of $K$. Then

$$
\lim _{n \rightarrow \infty} \frac{1}{\left|P_{g, n}\right|}\left(\sum_{K \in P_{g, n}} \sigma(K)\right)=2 g .
$$

(Note that $P_{g, n}$ is always finite, and becomes non-empty for fixed $g$ when $n$ is large enough. Note also that in general the crossing number $c(K)$ of a positive knot $K$ may not be admitted by a positive diagram, as shown in [39].)

This theorem means that generically the value of $\sigma$ for fixed genus is the maximal possible. From this point of view, the philosophy behind the Growth conjecture is that "when the generic value is the maximal possible, the minimal value should not be too small.' Theorem 1.4 is a consequence of a (largely unrelated to the subject of this paper) extension of the asymptotical denseness result for special alternating knots in [44], which is proved in a separate paper [36].

\subsection{Overview of the proof}

Consider the Growth conjecture in what follows for knots. In this subsection, before we get into considerable technicalities, we will give a summary of the difficulty in, and strategy for the proof of Theorem 1.3. (A few technical terms occurring will be explained in Section 2.)

For the proof we need a method to evaluate

$$
\min \{\sigma(K): K \text { positive, } g(K) \geq n\} \text {. }
$$

To do so, first we apply generator theory for canonical genus [38; 43; 44]. This allows to calculate

$$
\min \{\sigma(K): K \text { positive, } g(K)=g\}
$$

for any given $g$ by verifying $\sigma$ on finitely many knots. These knots, the "generators" of [38], can be algorithmically constructed. We will not get into details about this 
procedure here, since we discussed it extensively elsewhere. Briefly, there are three methods (in increasing order of efficiency): selecting knots from the alternating knot tables (more efficient for small crossing number), using maximal Wicks forms (as explained in [43], more efficient for high crossing number), and using thickenings of trivalent graphs and Hirasawa's algorithm [34].

Then we need to obtain an upper bound on the genus necessary to check for (3), which will be our main effort. It is possible that always

$$
\begin{aligned}
\min \{\sigma(K): K \text { positive, } g(K) & =g+1\} \geq \\
& \min \{\sigma(K): K \text { positive, } g(K)=g\},
\end{aligned}
$$

and then we would need to check just genus $n$. Unfortunately, we do not know how to prove (4) (or whether it is always true). If we add a ' -2 ' on the r.h.s., then the inequality follows easily from the fact that any genus $g+1$ diagram can be turned into a genus $g$ diagram by smoothing out a (proper) pair of crossings. However, the negative correction term continuously ruins the estimate with increasing genus, and thus makes it useless with regard to the Growth conjecture. We will thus be forced to avoid smoothings and work only with crossing changes.

Thus we need a lower bound on the genus of a positive diagram one can obtain by crossing switches from a given one. To find such a bound is considerably more difficult. We will show that the genus decreases at most by a linear factor, which we will be able to drop to $\frac{221}{41} \approx 5.39$ (Theorem 4.1). While for the mere existence of such a constant a part of the proofs can be simplified, even the value we attained with the extra effort is still too large to make our result practical. Still one can take practical advantage of the arguments we apply, and we will attempt to settle the problem $\Sigma_{4}=\{2,3,4\}$ at a later stage along these lines.

In the proof of the genus decreasing bound generator theory finds again its application. The first step of this proof uses an improvement of the estimate of the maximal crossing number of a generating diagram of given genus [44], given in [36]. In the next section we review the necessary tools.

Then we need an estimate of the minimal length of simplifying bridges/tunnels. What we like is to choose a certain piece of the strand in a positive diagram, to switch properly crossings on it so that it becomes a bridge or tunnel, and then shrink the bridge or tunnel by a wave move. In case the diagram has a clasp this is trivial, so all difficulty comes from the diagrams that have no such clasps.

Since we want after the shrinking the diagram to be positive, we must ensure the shrunk bridge/tunnel to have length 1 , ie, only one intersection with the rest of the diagram. In 
that case with the option between bridge and tunnel we can always achieve the new diagram to be positive.

So we must investigate how long a piece of the strand needs to be, so that we can shrink it to a one-crossing bridge/tunnel. This is very similar to what was done by myself and M Kidwell in [40]. There he proved, improving my original result, that if a knot diagram contains a bridge of length more than $1 / 3$ of its crossing number, this bridge can be rerouted (wave moved) to a smaller one. Here, however, we must work harder, because we want to know when such a bridge can be rerouted to one of a single crossing. What we basically show is that to have this much stronger condition, we can replace Kidwell's constant $1 / 3$ by $36 / 41$. This is the content of the Curve length lemma in Section 3.

Note that we could also seek a bridge that can be rerouted to length 0 , but we will see that even finding (a moderately short) one shrinkable to length 1 is difficult enough. If a bridge/tunnel shrinks to more than one crossing, to ensure one can keep all crossings positive, it is necessary to take account on the orientations of the strands intersecting the shortened bridge/tunnel; this seems virtually unfeasible, though. A bonus of using a bridge/tunnel of length 1 is also that we can apply our work to Taniyama's partial order, see Theorem 5.6.

\section{General preliminaries}

Here we recall several basic facts and notations.

\subsection{Miscellanea}

By $\lfloor n\rfloor$ we will mean the greatest integer not greater than $n$. By $\lceil n\rceil$ we will mean the smallest integer not smaller than $n$.

For a set $S$, the expressions $|S|$ and $\# S$ are equivalent and both denote the cardinality of $S$. In the sequel the symbol ' $\subset$ ' denotes a not necessarily proper inclusion.

'W.l.o.g.' abbreviates 'without loss of generality' and 'r.h.s.' (resp. 'l.h.s.') 'right handside' (resp. 'left hand-side').

\subsection{Link diagrams}

Definition 2.1 A crossing $p$ in a knot diagram $D$ is called reducible (or nugatory) if $D$ can be represented in the form

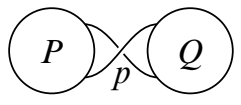

Algebraic 83 Geometric Topology, Volume 6 (2006) 
For a nugatory crossing $p$ in $D$ there is a curve in the plane intersecting $D$ only in $p$ (and doing so transversely). We call this curve the nugatory curve of $p$. A diagram $D$ is called reducible if it has a reducible crossing, else it is called reduced.

Definition 2.2 The diagram on the right of Figure 1 is called connected sum $A \# B$ of the diagrams $A$ and $B$. If a diagram $D$ can be represented as the connected sum of diagrams $A$ and $B$, such that both $A$ and $B$ have at least one crossing, then $D$ is called disconnected (or composite), else it is called connected (or prime).

Alternatively, if $D=A \# B$ then there is a closed curve $\beta$ in the plane intersecting $D$ in two points (and doing so transversely), such that $A$ and $B$ are contained in the in/exterior of $\beta$. We call $\beta$ a separating curve for $D$.

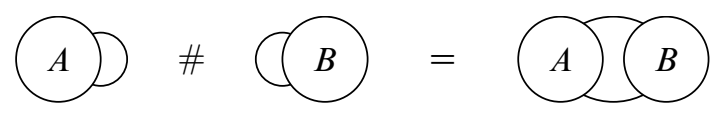

Figure 1

Note in particular that prime diagrams are reduced.

Definition 2.3 If there is a closed curve $\beta$ in the plane intersecting $D$ nowhere and containing at least one component of $D$ in both its interior and exterior, we say that $D$ is split and $\beta$ the splitting curve for $D$. A split component of a link $L$ is a maximal set $S$ of components of $L$ with the property that if $a, b \in S$, then in no split diagram $D$ of $L$ with $\beta$ as splitting curve, $a$ and $b$ land on different sides in $\mathbb{R}^{2} \backslash \beta$. A link is split if it has a split diagram, or equivalently, if it has more than one split component.

Consider 3 links differing just at one crossing.

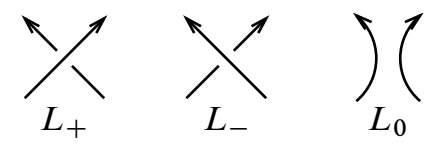

We call such a triple of links a skein triple.

A positive resp. negative crossing is the fragment of $L_{+}$resp. $L_{-}$shown in (5). Replacing any of these fragments by the fragment of $L_{0}$ in (5) is called smoothing out the crossing. The number of crossings of a diagram $D$ is written $c(D)$. The sum of signs of all crossings in $D$ is called writhe of $D$ and denoted by $w(D)$.

Smoothing out all crossings in $D$ one obtains a collection of loops in the plane called Seifert circles. We write $s(D)$ for the number of Seifert circles of a diagram $D$. 
A knot is called positive if it has a diagram with all crossings positive. Such a diagram is called also positive. A diagram is called $n$-almost positive, if it has exactly $n$ negative crossings. A positive diagram $D$ obtained from a diagram $D^{\prime}$ by crossing changes is called positification of $D^{\prime}$.

A diagram is called special if all its Seifert circles have empty interior or exterior. Such Seifert circles in an arbitrary diagram are called non-separating, the others are called separating. Any link diagram decomposes as the Murasugi sum (*-product) of special diagrams (see [8, Section 1]).

We call a Seifert circle $A$ opposite to another Seifert circle $B$ at a crossing $p$, if $p$ joins $A$ and $B$.

The (canonical) Euler characteristic $\chi(D)$ of a link diagram $D$ is defined as $\chi(D)=$ $s(D)-c(D)$, where $s(D)$ is, as before, the number of Seifert circles and $c(D)$ the number of crossings of $D$. If $D$ is a diagram of a link with $n$ components, the (canonical) genus $g(D)$ of $D$ is given by

$$
g(D)=\frac{2-n-\chi(D)}{2}=\frac{2-n+c(D)-s(D)}{2} .
$$

These are the genus and Euler characteristic of the canonical Seifert surface of $D$, the one obtained by applying Seifert's algorithm on $D$. The genus $g(L)$ and Euler characteristic $\chi(L)$ of a link $L$ are the minimal genus and maximal Euler characteristic of all Seifert surfaces of $L$, and the canonical genus $g_{c}(L)$ and canonical Euler characteristic $\chi_{c}(L)$ of $L$ are the minimal genus and maximal Euler characteristic of all canonical Seifert surfaces of $L$, ie, all Seifert surfaces obtained by applying Seifert's algorithm on some diagram $D$ of $L$.

The importance of the canonical genus relies on the following classical fact:

Theorem 2.4 For an alternating/positive knot or link $L$ with an alternating/positive diagram $D$ we have $g(D)=g(L)$. (In particular, for such knots or links canonical genus and ordinary genus coincide.)

In the alternating case this was proved by [10;22]. It can also be proved, in both cases, using [13]. For positive diagrams (and in particular positive braid representations) it follows from [9], or from Bennequin's inequality. The original form of this inequality is stated as follows.

Theorem 2.5 [2, theorem 3] If $\beta$ is a braid representation of a link $L$, then

$$
\chi(L) \leq n(\beta)-|[\beta]|,
$$


where $n(\beta)$ is the number of strands of $\beta$ and $[\beta]$ its algebraic crossing number (exponent sum).

This inequality admits several improvements. A first, and easy, observation is that by the braid algorithms of Yamada [50] and Vogel [48] we obtain a version for a general link diagram $D$ of $L$ :

$$
\chi(L) \leq s(D)-|w(D)| .
$$

We will use only this version of the inequality.

Later Rudolph [31;32] showed that the r.h.s. in Bennequin's inequality is actually an estimate for the (smooth) slice Euler characteristic.

$$
\chi_{s}(L) \leq s(D)-|w(D)| .
$$

This inequality was further extended by showing that one can replace the 1.h.s. with the invariants of Ozsváth-Szabó and Rasmussen on the one hand, and by slightly improving the r.h.s. on the other hand (adding a strongly negative Seifert circle term; see [32; 17]).

A clasp is a tangle made up of two crossings. According to the orientation of the strands we distinguish between reverse and parallel clasp.

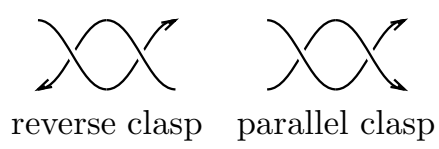

By switching one of the crossings in a clasp, one can eliminate the pair by a Reidemeister II move, and this procedure is called resolving the clasp.

Definition 2.6 A shadow of a link diagram is the plane curve of the diagram (ie, the object obtained by ignoring crossing information). A region of a link diagram $D$ is a connected component of the complement of the shadow of $D$. An edge of $D$ is the part of the plane curve of $D$ between two crossings (clearly each edge bounds two regions). A region is a bigon if it has only two corners. (A bigon in the shadow of $D$ corresponds to a clasp in $D$.)

At each crossing $p$, exactly two of the four adjacent regions contain a part of the Seifert circles near $p$. We call these the Seifert circle regions of $p$. The other two regions are called the non-Seifert circle regions of $p$. We call two regions opposite at a crossing $p$, if $p$ lies in the boundary of both regions, but they do not share any of the four edges bounded by $p$. If two regions share an edge, they are called neighbored.

A diagram has a (canonical up to interchanging colors) black-white region coloring, given by the condition that neighbored regions have different colors. This is called the checkerboard coloring. 
A bridge/tunnel ${ }^{3}$ of a link diagram is a piece of a strand passing exclusively through over/undercrossings. The number of such over/undercrossings is the length of the bridge/tunnel.

In certain situations, there is a move, called wave move, that allows to shrink a bridge/tunnel to one of a smaller length (see Figure 2, or also [40] for example).
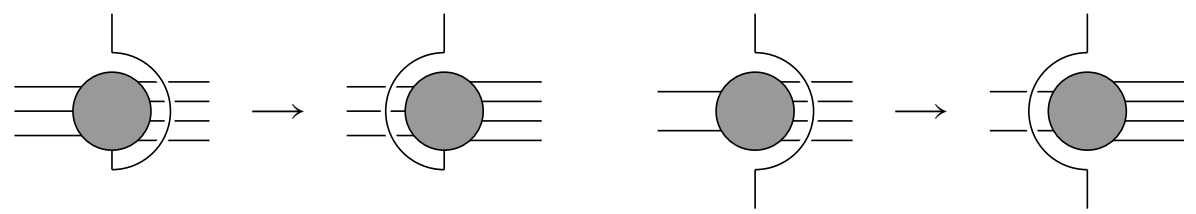

Figure 2: Wave-moves. The number of strands on left and right of the shaded circle may vary. It is only important that the parities are equal resp. different, and that the left-outgoing strands are fewer that the right-outgoing ones.

\subsection{The signature}

The signature $\sigma$ is a $\mathbb{Z}$-valued invariant of knots and links. It has several definitions. The most common one is using Seifert surfaces and linking pairings. See, eg, [29]. In the sequel, it will be more convenient to follow a rather different approach, using properties of the behaviour of $\sigma$ under local (diagram) transformations.

The Alexander polynomial $\Delta_{L}(t)$ can be specified by the relation

$$
\Delta\left(L_{+}\right)-\Delta\left(L_{-}\right)=\left(t^{1 / 2}-t^{-1 / 2}\right) \Delta\left(L_{0}\right),
$$

with $L_{ \pm, 0}$ as in (5), and the value 1 on the unknot. The signature $\sigma(L)$ is related a value of $\Delta$, called determinant, $\operatorname{det}(L)=\left|\Delta_{L}(-1)\right|$. We have that $\sigma(L)$ has the opposite parity to the number of components of a link $L$, whenever $\Delta_{L}(-1) \neq 0$. This in particular always happens for $L$ being a $\operatorname{knot}\left(\Delta_{L}(-1)\right.$ is always odd in this case), so that $\sigma$ takes only even values on knots.

Most of the early work on the signature was done by Murasugi [24], who showed several properties of this invariant. If $L_{+}, L_{-}$and $L_{0}$ form a skein triple, as in (5), then ${ }^{4}$

$$
\begin{aligned}
& \sigma\left(L_{+}\right)-\sigma\left(L_{-}\right) \in\{0,1,2\} \\
& \sigma\left(L_{ \pm}\right)-\sigma\left(L_{0}\right) \in\{-1,0,1\} .
\end{aligned}
$$

\footnotetext{
${ }^{3}$ The term 'tunnel' has usually a different meaning in knot theory. We nevertheless use it here in this meaning, since it is suggestive, and the other (classical) sort of tunnels never occur in this paper.

${ }^{4}$ Keep in mind that our sign choice of $\sigma$ follows [30] and is different from [24].
} 
Further, Murasugi found the following important relation between $\sigma(K)$ and $\operatorname{det}(K)$ for a knot $K$.

$$
\begin{aligned}
& \sigma(K) \equiv 0(4) \Longleftrightarrow \operatorname{det}(K) \equiv 1(4) \\
& \sigma(K) \equiv 2(4) \Longleftrightarrow \operatorname{det}(K) \equiv 3(4)
\end{aligned}
$$

These conditions, together with the initial value $\sigma(\bigcirc)=0$ for the unknot, and the additivity of $\sigma$ under split union (denoted by ' $\sqcup$ ') and connected sum (denoted by '\#')

$$
\sigma\left(L_{1} \# L_{2}\right)=\sigma\left(L_{1} \sqcup L_{2}\right)=\sigma\left(L_{1}\right)+\sigma\left(L_{2}\right),
$$

allow one to calculate $\sigma$ for very many links. In particular, if we have a sequence of knots $K_{i}$

$$
K_{0} \rightarrow K_{1} \rightarrow K_{2} \cdots \rightarrow K_{n}
$$

such that $K_{n}$ is the unknot and $K_{i}$ differs from $K_{i-1}$ only by one crossing change, then (7) and (9) allow to calculate inductively $\sigma\left(K_{i}\right)$ from $\sigma\left(K_{i+1}\right)$, if $\operatorname{det}\left(K_{i}\right)$ is known.

From this the following property is evident for knots, which also holds for links: $\sigma(! L)=-\sigma(L)$, where $! L$ is the mirror image of $L$.

\subsection{Genus generators}

Consider the set of alternating knots $K$ of genus $g(K)=g$ and crossing number $c(K)=n$. This set was shown to have special structure by a theorem of [38], discovered independently and simultaneously by M Brittenham [4]. In order to state this theorem, we start with some classical definitions.

By the work of Menasco and Thistlethwaite [21], alternating knots are intimately related to a diagrammatic move called flype.

Definition 2.7 A flype is a move on a diagram shown in Figure 3.
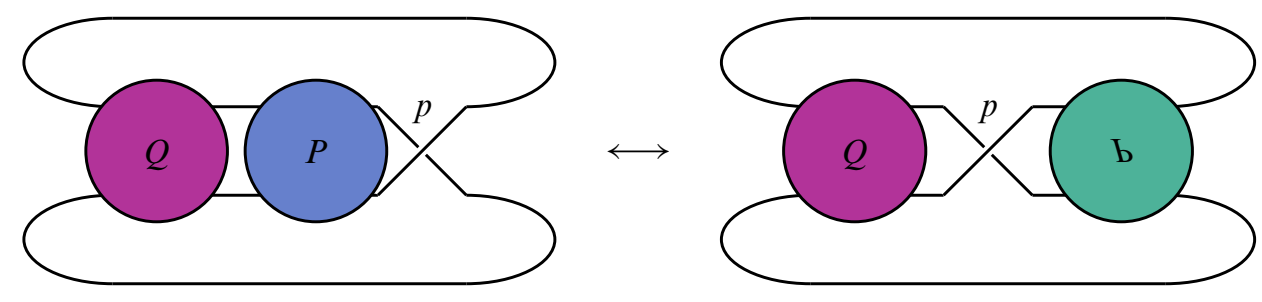

Figure 3: A flype near the crossing $p$ 
When we want to specify the distinguished crossing $p$, we say that it is a flype near the crossing $p$.

The tangle $P$ on Figure 3 we call flypable, and we say that the crossing $p$ admits a flype or that the diagram admits a flype at (or near) $p$. The crossing $p$ is called flype crossing.

Definition 2.8 A primitive Conway tangle [7] is a tangle of the form
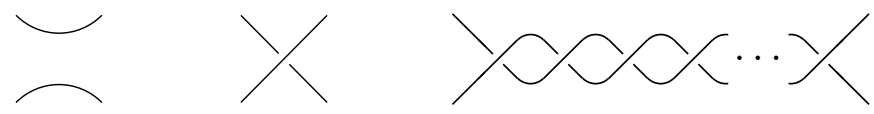

Two crossings of a diagram are twist equivalent if they are contained in a primitive Conway tangle.

We call the flype in Figure 3 non-trivial, if both tangles $P$ and $Q$ have crossings not twist equivalent to the flype crossing (in particular they have both at least two crossings).

Since trivial flypes are of no interest we will consider all subsequent flypes to be non-trivial, without mentioning this explicitly each time, unless otherwise noted.

Theorem 2.9 [38] Reduced (that is, with no nugatory crossings) alternating knot diagrams of given genus decompose into finitely many equivalence classes under flypes and (reversed) applications of antiparallel twists at a crossing:

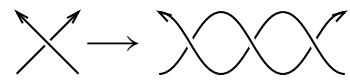

Henceforth we call the move in (10) a $\vec{t}_{2}$ move.

It was observed in [38] that in a sequence of flypes and $\vec{t}_{2}$ moves, all the flypes can be performed in the beginning. It follows then from [21] that there are only finitely many alternating knots with $\vec{t}_{2}$-irreducible diagrams of given genus $g$, and we call all such knots, and their alternating diagrams generators or generating knots/diagrams of genus $g$. The positifications of generating diagrams of genus $g$ are called positive generating diagrams.

There is an obvious bijective correspondence between the crossings of the 2 diagrams in Figure 3 before and after the flype, and under this correspondence we can speak of what is a specific crossing after the flype. In this sense, we make the following definition: 
Definition 2.10 We call two crossings in a diagram $\sim$-equivalent, if they can be made to form a reverse clasp after some (sequence of) flypes.

It is an easy exercise to check that $\sim$ is an equivalence relation, and that two crossings are $\sim$-equivalent iff they share the same pair of non-Seifert circle regions.

Definition 2.11 We call an alternating diagram generating, or a generator, if each $\sim$ equivalence class of its crossings has 1 or 2 elements. The set of diagrams which can be obtained by applying flypes and $\vec{t}_{2}$ moves on a generating diagram $D$ we call (generating) series of $D$.

Thus Theorem 2.9 says that alternating diagrams of given genus decompose into finitely many generating series.

Definition 2.12 Let $c_{g}$ be the maximal crossing number of a generating diagram of genus $g$, and $d_{g}$ the maximal number of $\sim$-equivalence classes of such a diagram.

\section{The curve length lemma}

In the following we will sometimes for convenience identify a diagram and its shadow (its plane curve with transverse self-intersections).

Let $\gamma$ be a curve, a piece of the solid line of a prime diagram shadow $D$. Curves are considered up to homotopy preserving the order of edges they pass, and transversality of their intersections. The complement $D \backslash \gamma$ of a curve is a planar graph with 4-valent vertices, with two exceptional vertices being of valence 1 . Let $v_{1,2}$ be those vertices, the start and end of $\gamma$ (we think of $\gamma$ as going from $v_{1}$ to $v_{2}$ ). For $D \backslash \gamma$ regions and edges can be defined analogously as for $D$.

Let the length len $\gamma$ of a curve $\gamma$ be the number of its intersections with the rest of the diagram, or the number of regions it passes (start and end region included, and possibly reentered regions counted multiply) minus one.

Definition 3.1 Let start and end $v_{1,2}$ of $\gamma$ lie in neighbored regions in $D \backslash \gamma$ (that is, $\gamma$ can be rerouted, or wave-moved, to a curve of one crossing), and len $\gamma>1$. Then we call $\gamma$ admissible. If $\gamma$ has minimal length among all admissible curves we call $\gamma$ minimal admissible.

We assume for the rest of this section, unless noted otherwise, that

$\gamma$ is a minimal admissible curve and $D$ has no bigon regions (clasps) and is prime.

Under these conditions we have several lemmas.

Algebraic 83 Geometric Topology, Volume 6 (2006) 
Lemma 3.2 A minimal admissible curve $\gamma$ passes any region $X$ of $D \backslash \gamma$ at most once (ie, $X \cap \gamma$ is empty or a single arc).

Proof Assume $\gamma$ passes a region $X$ of $D \backslash \gamma$ twice, and let $x_{1,2}$ be points in the interior of $X$ on $\gamma$ in order of passing. Let $\tilde{\gamma}$ be the part of $\gamma$ between $x_{1,2}$. By the checkerboard coloring, $\tilde{\gamma}$ has even length, and by assumption, this length is non-zero. If $\tilde{\gamma}$ has length 2 , then either $D$ is composite, or has a bigon region (clasp), which we excluded. So $\tilde{\gamma}$ has length at least 4 . Then taking the part of $\gamma$ starting in the region after $x_{1}$ till $x_{2}$ gives a shorter admissible curve $\gamma$.

Apply the transformation (11) or (12) to get a diagram shadow $D^{\prime}$ with a curve $\gamma^{\prime}$ starting and ending on edges of $D^{\prime}$.
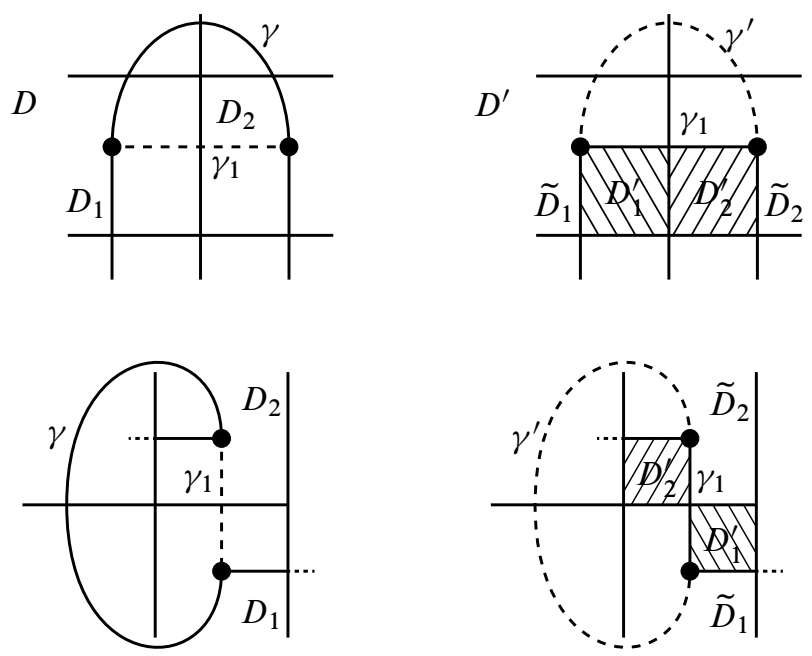

Let $\gamma_{1}$ be a one crossing curve connecting the start and end of $\gamma$ in $D$. This curve $\gamma_{1}$ is determined uniquely by the edge is passes, but this edge may not be unique. Thus $\gamma_{1}$ is not uniquely determined in general. It will be useful to remark that $\gamma_{1}$ is unique if $D^{\prime}$ is prime, and otherwise there are at most two possible $\gamma_{1}$. If ambiguous, fix some particular choice of $\gamma_{1}$. Then let $D^{\prime}=(D \backslash \gamma) \cup \gamma_{1}$. (We denote by $\gamma_{1}$ the same arc in $D$ and $D^{\prime}$, while we distinguish between $\gamma^{\prime}$ and $\gamma$; so $\gamma^{\prime}$ in $D^{\prime}$ is the "trace" of $\gamma$ in $D$.) We can assume w.l.o.g. that $\gamma$ and $\gamma_{1}$ do not intersect (in interior points), otherwise a shorter pair of curves will also do.

$D^{\prime}$ has two specific regions $D_{1,2}^{\prime}$ which can be described by the property that they are bounded by a piece of $\gamma_{1}$, but do not contain a piece of $\gamma^{\prime}$ at its start or end. Let $\widetilde{D}_{1,2}$ be the regions of $D^{\prime}$ containing the start and end of $\gamma^{\prime}$ and $D_{i}=D_{i}^{\prime} \cup \widetilde{D}_{i}$. The $D_{i}$ can (and will) be regarded also as regions of $D \backslash \gamma$. 
The following lemmas are suggestive from the diagrams in (11) and (12), but they are heavily applied in the following arguments and still require a bit of proof.

Lemma 3.3 The crossing on $\gamma_{1}$ in $D^{\prime}$ is not nugatory. In other words, the four regions $D_{i}^{\prime}, \widetilde{D}_{i}$ for $i=1,2$ in $D^{\prime}$ are all pairwise distinct.

Proof We prove indirectly. Let this crossing, call it $x$, be nugatory, and $\delta^{\prime}$ be a nugatory curve for $x$ in $D^{\prime}$, and $\delta$ its preimage in $D$. (We write $x$ also for the preimage of $x$ in $D$.) Since $\delta^{\prime}$ intersects $D^{\prime}$ only in $x$, the start and end of $\gamma_{1}$ must lie in different regions of $\mathbb{R}^{2} \backslash \delta$. Therefore, $\left|\delta^{\prime} \cap \gamma^{\prime}\right|=|\delta \cap \gamma|$ is odd. Now, as $\delta^{\prime}$ is a nugatory curve, $\delta$ passes only one region of $D \backslash \gamma$. Using Lemma 3.2, and a proper homotopy of $\delta^{\prime}$, we see that we can reduce the intersections between $\delta$ and $\gamma$ to one. We assumed that $\gamma \cap \gamma_{1}=\varnothing$, so that $x \notin \gamma^{\prime}$, and so we can choose the homotopy so that $\delta^{\prime}$ is still a nugatory curve for $x$. Now, since $\delta \cap(D \backslash \gamma)=\{x\}$ and $|\delta \cap \gamma|=1$, we have $|\delta \cap D|=2$. By primality of $D$, (after pushing $\delta$ off $x$ slightly) one of the regions of $\mathbb{R}^{2} \backslash \delta$ contains just a trivial arc of $D$. But this arc must then contain one of the endpoints of $\gamma$ and $\gamma_{1}$. Also, the strand of $D \backslash \gamma$ that intersects $\delta$ in $x$ must continue into this arc. So $x$ must be an intersection of $\gamma_{1}$ with $\gamma$, which we excluded. This is a contradiction.

Lemma 3.4 $\gamma^{\prime}$ does not enter into $D_{1}^{\prime}$ or $D_{2}^{\prime}$, ie, $\gamma^{\prime} \cap\left(\right.$ int $D_{1}^{\prime} \cup$ int $\left.D_{2}^{\prime}\right)=\varnothing$. (Here 'int' stands for the topological interior.)

Proof By construction the initial and terminal parts of $\gamma^{\prime}$ do not enter into $D_{1}^{\prime}$. Also $\gamma^{\prime} \cap \gamma_{1}=\varnothing$ by assumption. Now let $e_{1}$ be the edge of the shadow of $D^{\prime} \backslash \gamma_{1}$ that contains the start point $v_{1}$ of $\gamma^{\prime}$ (and $\gamma_{1}$ ). If $\gamma^{\prime}$ intersects $e_{1}$ in $D^{\prime}$ before leaving $D_{1}=\widetilde{D}_{1} \cup D_{1}^{\prime}$, then $D$ is not prime, which we excluded. Since $\gamma^{\prime} \cap \gamma_{1}=\varnothing$, this means that $\gamma^{\prime}$ must enter $D_{1}^{\prime}$ through an edge which lies in the boundary of $D_{1}$ in $D$. Then $\gamma$ passes $D_{1}$ in $D \backslash \gamma$ twice, in contradiction to Lemma 3.2. The case of $D_{2}^{\prime}$ is similar.

Lemma 3.5 The only neighbored regions to $D_{1,2}^{\prime}$ passed by $\gamma^{\prime}$ are $\widetilde{D}_{1,2}$.

Proof Let, contrarily, w.l.o.g. $X$ be a neighbored region to $D_{1}^{\prime}$ in $D^{\prime}$, different from $\widetilde{D}_{1,2}$, that contains a part of $\gamma^{\prime}$. Then $X$ does not contain an initial or terminal arc of $\gamma^{\prime}$. So taking the part $\hat{\gamma}^{\prime}$ of $\gamma^{\prime}$ from $v_{1}$ to (an interior point of) $X$ would give a shorter admissible curve $\hat{\gamma}$ in $D$, unless len $D \hat{\gamma}=1$.

Assume len ${ }_{D} \hat{\gamma}=1$. Since $\hat{\gamma} \cap \gamma_{1} \subset \gamma \cap \gamma_{1}=\varnothing$, then also len $D^{\prime} \hat{\gamma}^{\prime}=1$. Now the curve $\hat{\gamma}^{\prime}$ starts at $v_{1}$ in $D^{\prime}$, and then enters either $D_{1}^{\prime}$ or $\widetilde{D}_{1}$. Since $\widetilde{D}_{1}$ has the same 
checkerboard color as $X$ in $D^{\prime}$, the curve $\hat{\gamma}^{\prime}$ needs at least two edges to intersect to reach $X$ from $\widetilde{D}_{1}$. Therefore, if len $D^{\prime} \hat{\gamma}^{\prime}=1$, then $\hat{\gamma}^{\prime}$ enters $D_{1}^{\prime}$. Then the same is true, however, for $\gamma^{\prime} \supset \hat{\gamma}^{\prime}$, but this contradicts Lemma 3.4.

This contradiction shows that len $D \hat{\gamma}=\operatorname{len}_{D^{\prime}} \hat{\gamma}^{\prime}>1$. So $\hat{\gamma}$ is admissible in $D$, and again we have a contradiction to the minimality of the admissible curve $\gamma$.

Lemma 3.6 There exists a unique edge of $D^{\prime}$, the one containing $v_{j}$, that bounds (simultaneously) $\widetilde{D}_{j}$ and $D_{j}^{\prime}$ (for $j=1,2$ ). If $\widetilde{D}_{j}$ and $D_{3-j}^{\prime}$ are neighbored in $D^{\prime}$, then, again, their commonly bounding edge is unique.

Proof Consider w.l.o.g. only $j=1$. Let $g_{i}$ be the parts of $\gamma_{1}$ in $D^{\prime}$ that bound $D_{i}^{\prime}$ and $\widetilde{D}_{i}$ for $i=1,2$. Let $e_{i}$ be the edge of $D^{\prime} \backslash \gamma_{1}$ that contains $v_{i}$ (or bounds $D_{i}$ on either side). Clearly $x_{1}=g_{1} \cup e_{1}$ is an edge in $D^{\prime}$ that bounds $D_{1}^{\prime}$ and $\widetilde{D}_{1}$. Assume there is another such edge $x_{1}^{\prime}$. Then ( $D^{\prime}$ is composite and) there is a separating curve $\delta$ in $D^{\prime}$ that intersects $D^{\prime}$ only in $x_{1}$ and $x_{1}^{\prime}$. By homotopy we can assume w.l.o.g. that $\delta$ intersects $x_{1}=g_{1} \cup e_{1}$ in some point on $e_{1}$, so $\delta \cap g_{1}=\varnothing$. Now, since $\delta$ passes only the regions $D_{1}^{\prime}$ and $\widetilde{D}_{1}$, by Lemma $3.3, \delta$ cannot intersect $g_{2}$. So $\delta \cap\left(g_{1} \cup g_{2}\right)=\delta \cap \gamma_{1}=\varnothing$. Then $v_{1,2}$ lie in the same region of $\mathbb{R}^{2} \backslash \delta$.

Now consider $\delta \cap \gamma^{\prime}$. Since $\delta$ passes only $D_{1}^{\prime}$ and $\widetilde{D}_{1}$, but $\gamma^{\prime}$ does not enter $D_{1}^{\prime}$, we have $\delta \cap \gamma^{\prime} \subset \widetilde{D}_{1}$. Now both $\gamma^{\prime}$ and $\delta$ have only one arc in $\widetilde{D}_{1}$ (for $\gamma^{\prime}$ use Lemma 3.2), so by homotopy we may assume that $\left|\delta \cap \gamma^{\prime}\right| \leq 1$. But since $\delta \cap \gamma_{1}=\varnothing$ and $\left|\delta \cap\left(\gamma^{\prime} \cup \gamma_{1}\right)\right|$ is even (by Jordan curve), we see $\delta \cap\left(\gamma^{\prime} \cup \gamma_{1}\right)=\varnothing$. Then, however, $\delta$ remains a separating curve in $D$, and $D$ is composite, a contradiction.

This shows the lemma for an edge bounding $D_{1}^{\prime}$ and $\widetilde{D}_{1}$. The argument for (an edge between) $D_{2}^{\prime}$ and $\widetilde{D}_{1}$ is similar.

The following is useful to record here, though it will be needed only in a later stage.

Lemma 3.7 $\gamma^{\prime}$ does not pass a region twice in $D^{\prime}$, and len $\gamma^{\prime}=\operatorname{len} \gamma$.

Proof The only difference between the regions of $D \backslash \gamma$ and $D^{\prime}$ is that $D_{i}$ are subdivided into $D_{i}^{\prime}$ and $\widetilde{D}_{i}$. However, by Lemma $3.4, \gamma^{\prime}$ does not pass $D_{i}^{\prime}$, so the claim follows from Lemma 3.2.

Let $r\left(D^{\prime}\right)$ be the number of regions of $D^{\prime}$. The main content of this section, whose proof will occupy its rest, is the following estimate. 
Lemma 3.8 (Curve length lemma) Assume again that $D$ is prime and that it has no clasp. Then $\gamma^{\prime}$ passes

$$
\text { len } \gamma^{\prime}+1 \leq \max \left(\frac{36 r\left(D^{\prime}\right)-96}{41}, 4\right)
$$

regions of $D^{\prime}$, including first and last.

Proof We will again for convenience abuse the distinction between the shadow $D$ and the positive diagram realizing it. Take a prime diagram $D$, and choose $\gamma$ with start and end in neighbored regions of $D \backslash \gamma$, which has length $>1$. Such a curve always exists. For example, take a loop and consider the curve going from the loop crossing until before the last crossing the loop passes.

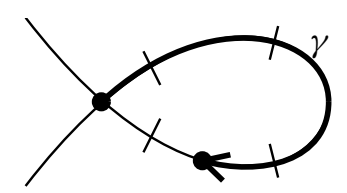

Clearly start and end region of such a curve are neighbored. If the curve has length 1 , then $D$ is composite or has a clasp, in contradiction to our assumption.

We fix now among all admissible $\gamma$ (not necessarily such that come from a loop) one of minimal length $(>1)$, and the (chosen, if ambiguous) curve $\gamma_{1}$ of one crossing connecting start and end of $\gamma$.

When $D$ is prime, often $D^{\prime}$ will also be prime. We want to show that $D^{\prime}$ becomes composite only in a very restricted situation.

Assume that $D^{\prime}$ is not prime. Let $\beta^{\prime}$ be a separating curve of $D^{\prime}$. (Recall that $\beta^{\prime}$ is characterized by intersecting $D^{\prime}$ in two points, and its interior and exterior being not simple arcs.) Thus $\beta^{\prime}$ passes exactly two regions of $D^{\prime}$. If a region $X$ in $D^{\prime}$ is passed by some separating curve $\beta^{\prime}$, then we call $X$ a separating region.

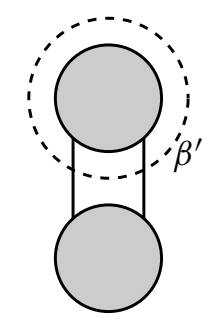

Sublemma 3.9 $D^{\prime}$ has at most one pair of separating regions, and if so, they are the start and end region of $\gamma^{\prime}$.

Algebraic $8 \mathcal{G}$ Geometric Topology, Volume 6 (2006) 
Proof Fix a separating curve $\beta^{\prime}$ in $D^{\prime}$. It has a preimage in $D$ we call $\beta$. This curve $\beta$ cannot be a separating curve of $D$ by primeness assumption. So the separating property must have been spoiled when recovering $D$ from $D^{\prime}$. If $\beta$ does not intersect $\gamma \cup \gamma_{1}$ in $D$, then the move from $D$ to $D^{\prime}$ must eliminate all crossings inside or outside of $\beta$, which clearly does not happen.

Thus $\beta$ must intersect $\gamma \cup \gamma_{1}$ in $D$. It intersects $\gamma \cup \gamma_{1}$ in some non-zero even number of points, and $D \cup \gamma_{1}$ in exactly two other points not on $\gamma$.

Assume now $\beta$ intersects $\gamma_{1}$ in $D$. Then $\beta^{\prime}$ does so also in $D^{\prime}$, that is, (at least) one of the two points on $\beta^{\prime} \cap D^{\prime}$ lies on $\gamma_{1}$.

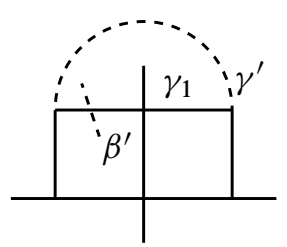

If $\beta^{\prime}$ intersects $\gamma_{1}$ twice, then by Lemma 3.3 it must do so on the same side of the crossing of $\gamma_{1}$ in $D^{\prime}$. (Keep in mind that $\beta^{\prime}$ passes only two regions in $D^{\prime}$.) Then, however, since $\beta^{\prime}$ intersects $D^{\prime}$ in no further points, it cannot be a separating curve. Thus $\beta^{\prime}$ intersects $\gamma_{1}$ only once, and $\gamma^{\prime}$ at least once (so that also $\beta \cap \gamma \neq \varnothing$ ).

Then $\beta^{\prime}$ passes through one of the regions $D_{1,2}^{\prime}$, which do not contain a part of $\gamma^{\prime}$. By Lemma 3.5, the only neighbored regions to $D_{1,2}^{\prime}$ containing a part of $\gamma^{\prime}$ in $D^{\prime}$ are $\widetilde{D}_{1,2}$. But if $\beta^{\prime}$ passes only through $D_{i}^{\prime}$ and $\widetilde{D}_{i}$ in $D^{\prime}$, then $\beta$ remains in $D$ entirely within $D_{i}$. Consequently, one of the interior or exterior of $\beta^{\prime}$ in $D^{\prime}$ contains only a trivial arc, a contradiction.

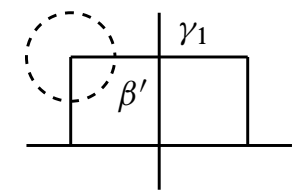

If $\beta^{\prime}$ passes through $D_{i}^{\prime}$ and $\widetilde{D}_{3-i}$, then we could have something like:

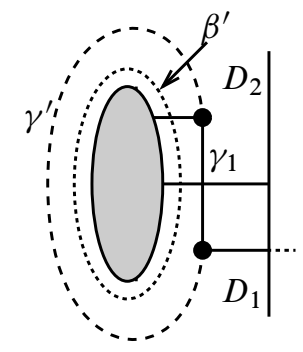

Algebraic ${ }^{6} \mathcal{G}$ Geometric Topology, Volume 6 (2006) 
However, we assumed that $\beta$ (and so $\beta^{\prime}$ ) intersects $\gamma_{1}$. Then $\beta^{\prime}$ would also pass through $\widetilde{D}_{i}$ or $D_{3-i}^{\prime}$. By Lemma 3.3, this will be a third region $\beta^{\prime}$ passes, again a contradiction.

Therefore, $\beta$ does not intersect $\gamma_{1}$ in $D$. Thus $\beta$ intersects non-trivially $\gamma$ in $D$ (and $\beta^{\prime}$ intersects $\gamma^{\prime}$ in $D^{\prime}$ ) in an even number of points, and two other points of $D$ not in $\gamma$. Additionally, we see that $\beta^{\prime}$ does not pass $D_{1,2}^{\prime}$. Otherwise, it would have to pass through a neighbored region of $D_{1,2}^{\prime}$ containing a part of $\gamma^{\prime}$. The only such regions are $\widetilde{D}_{1,2}$, and then we have a contradiction using Lemma 3.6 and the preceding argument.

Since $\beta^{\prime}$ passes through exactly two regions in $D^{\prime}=(D \backslash \gamma) \cup \gamma_{1}$, its preimage $\beta$ can pass through at most two regions in $D \backslash \gamma$. If it passes through only one region in $D \backslash \gamma$, then it must be some of the $D_{i}$, which are subdivided in $D^{\prime}$. However, then $\beta^{\prime}$ passes in $D^{\prime}$ through $D_{i}^{\prime}$, which we argued out. Thus $\beta$ passes through exactly two different regions $X$ and $Y$ in $D \backslash \gamma$, which are therefore neighbored in $D \backslash \gamma$.

We claim that $\beta$ cannot intersect the two points in $D \backslash \gamma$ consecutively, without intersecting $\gamma$ in between. Otherwise, all intersections of $\beta$ with $\gamma$ would be consecutive, say in region $X$ of $D \backslash \gamma$.
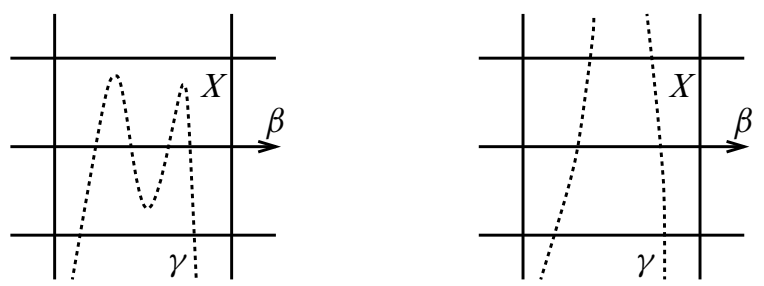

Since by Lemma 3.2, $\gamma$ does not reenter the same region $X$ (ie, the second of the above two pictures does not occur), we see (as in the first of the above pictures) that we can homotope $\beta$ within $X$ off $\gamma$, that is, we can find a curve $\tilde{\beta}$ in $D$ not intersecting $\gamma$, such that in $D^{\prime}$ we still have the form (14). This is a contradiction to the preceding arguments that $\gamma$ intersects $\beta$.

Since $\beta$ intersects $\gamma$ between the two intersections with $D \backslash \gamma$, this means also that both $X$ and $Y$ are passed by $\gamma$. Assume some of $X$ and $Y$ is not the start or end region of $\gamma$ in $D \backslash \gamma$. Then by the minimality of $\gamma$ and Lemma 3.2, $\gamma$ must pass directly from $X$ to $Y$. By primeness of $D$, there is a unique edge of $D \backslash \gamma$ to pass to move (directly) from $X$ to $Y$. (See the remark below (12).) So we see that $\beta$ and $\gamma$ pass from $X$ to $Y$ through the same edge of $D \backslash \gamma$. Since this edge is clearly not intersected by $\gamma_{1}$, then we can homotope $\beta^{\prime}$ in $D^{\prime}$ so that $\beta$ and $\gamma$ do not intersect in $X$. Thus $\beta$ intersects $\gamma$ only in $Y$, and then the intersections of $\beta$ with $D \backslash \gamma$ are consecutive, which we argued out. 
So the two regions $X$ and $Y$ are the start and end regions of $\gamma$ in $D \backslash \gamma$, which we called $D_{1,2}$. When turning $D$ into $D^{\prime}$, each of $D_{1,2}$ are subdivided into two parts by a piece of $\gamma_{1}$. These parts are $D_{1,2}^{\prime}$ and $\widetilde{D}_{1,2}$. We argued that $\beta^{\prime}$ does not pass $D_{1,2}^{\prime}$. Thus it intersects the other two parts $\widetilde{D}_{1,2}$. By a checkerboard coloring argument, we see that we must have the case (11) (rather than (12)), and $D$ looks like:

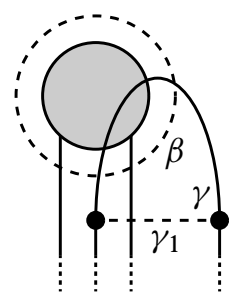

So the start and end region $\widetilde{D}_{1,2}$ of $\gamma^{\prime}$ are the only regions passed by $\beta^{\prime}$. The proof of Sublemma 3.9 is therefore complete.

We wish to bound from below now the number of regions of $D^{\prime}$ in terms of len $\gamma^{\prime}$. For this sake, we will count the regions of $D^{\prime}$ "close" to $\gamma^{\prime}$.

Number the regions of $D^{\prime}$ and make a table (Figure 4). This table contains two columns, left and right, and a row for each intermediate region $R$ (ie, $R \neq \widetilde{D}_{1,2}$ ) passed by $\gamma^{\prime}$. Note that the number of regions $R$ is non-zero by assumption of admissibility on $\gamma$. We call a part of the table given by the row and choice of left/right side a slot.

For each region $R$ passed by $\gamma^{\prime}$, except first and last, order into the left and right side of the table the neighbored regions $R^{\prime}$ to $R$ from left/right of $\gamma^{\prime}$ in negative/positive rotation sense, as shown in the below figure. These are the regions neighbored to $R$ by edges not intersected by $\gamma^{\prime}$. The edges separating $R$ and $R^{\prime}$ are unique for any pair of neighbored regions $R, R^{\prime}$ because we proved in Sublemma 3.9 that any separating curve of $D^{\prime}$ does not pass through a region $R$ passed intermediately by $\gamma^{\prime}$.

In the row of the table corresponding to $R$, put the regions on the left/right of $\gamma^{\prime}$ thus ordered from bottom to top.

Then each neighbored region $R^{\prime}$ entered into the table is not passed by $\gamma^{\prime}$, otherwise there is a contradiction to the minimality of its length. For the same reason, using our preparatory lemmas, we showed that $D_{1,2}^{\prime}$ are not passed by $\gamma^{\prime}$, and also any neighbored region of theirs is not passed by $\gamma^{\prime}$ as an intermediate region (ie, not first or last). Thus $D_{1,2}^{\prime}$ do not occur in the table.

We would like to count the regions $R^{\prime}$ in the table, but must avoid duplications. We will thus make some effort to bound the number of such duplications.

For this we remark that there are several rules the entries in the table satisfy: 

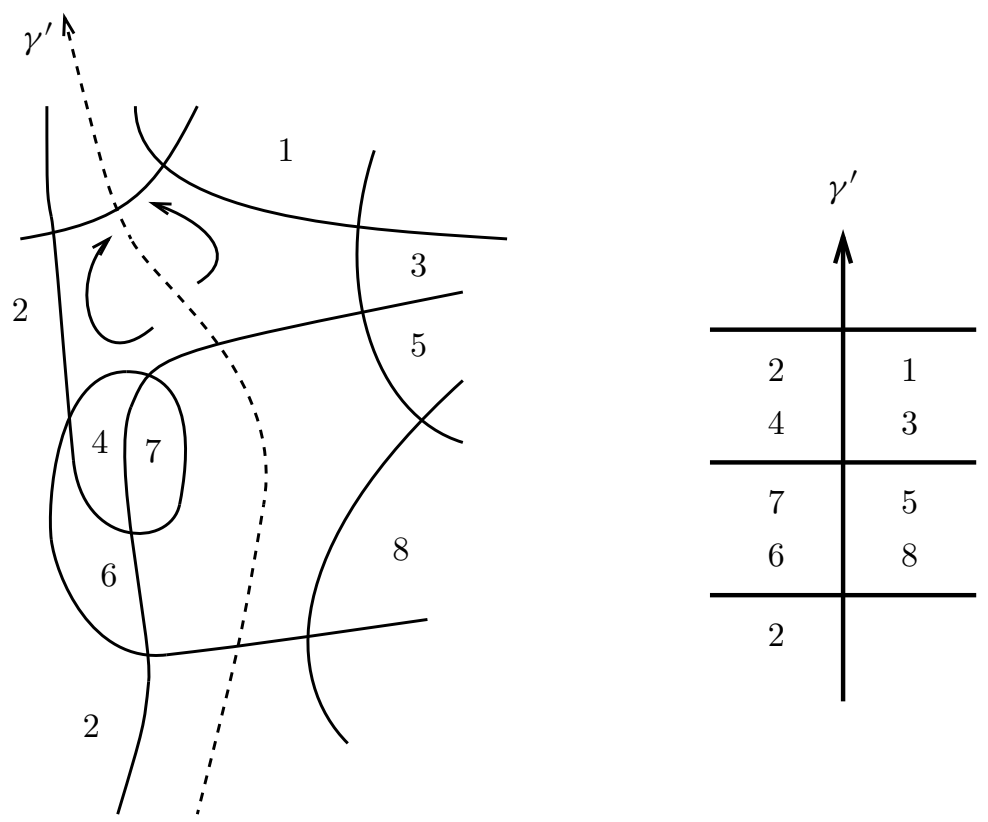

Figure 4

- All entries on the left and right on the same line are distinct (because the restriction to the separating region pairs in $D^{\prime}$ ).

- All entries on the left are distinct from all entries on the right (in whatever row). This follows from the Jordan curve theorem applied on $\gamma \cup \gamma_{1}$.

- Each row contains at least one entry (on the left or right), ie, there are no empty rows:

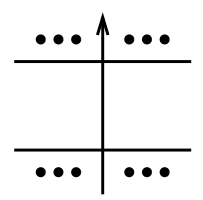

Otherwise we have a move

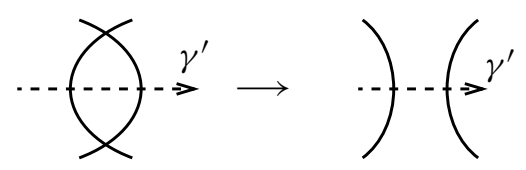

Algebraic 83 Geometric Topology, Volume 6 (2006) 
which shrinks an admissible curve $\gamma$ of length 3 in $D$, and since by Lemma 3.7, len $\gamma^{\prime}=$ len $\gamma=3$, we are done, using the second alternative in the maximum in (13).

- There are no two consecutive rows which are empty on the same side, ie:

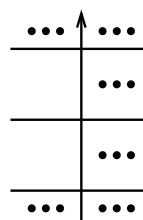

or

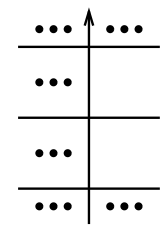

Otherwise we can find a shorter curve $\gamma$ of length 3 , which would shrink to $\gamma_{1}$ like

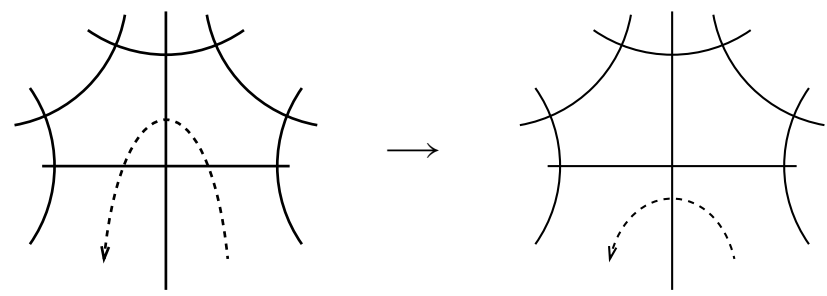

and again are done using the second alternative in the maximum in (13). Finally,

- there are no 3 consecutive rows with only one entry (left or right):

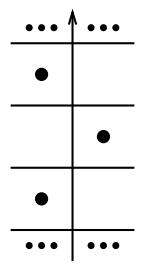

or

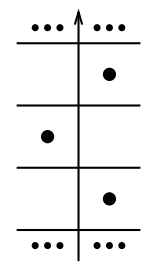

(The previous point rules out the other patterns.) Otherwise we have a picture like this:
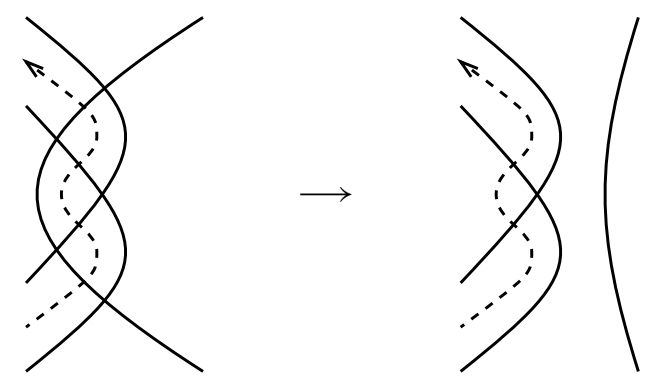

Algebraic 83 Geometric Topology, Volume 6 (2006) 
The right strand then contains a curve $\gamma^{\prime}$ (or $\gamma$ in $D$ ) of length 3 , and so we are done as in the previous point.

Replace now in table numbers by beads, and introduce an equivalence relation between beads coming from the same number (=region). If now two beads are equivalent, then they have the same parity of row (because of the checkerboard coloring), and the same side (because of the Jordan curve theorem applied on $\gamma \cup \gamma_{1}$ ).

Join by an arc two consecutive equivalent beads on left or right (ie, all beads between them are not equivalent to them).
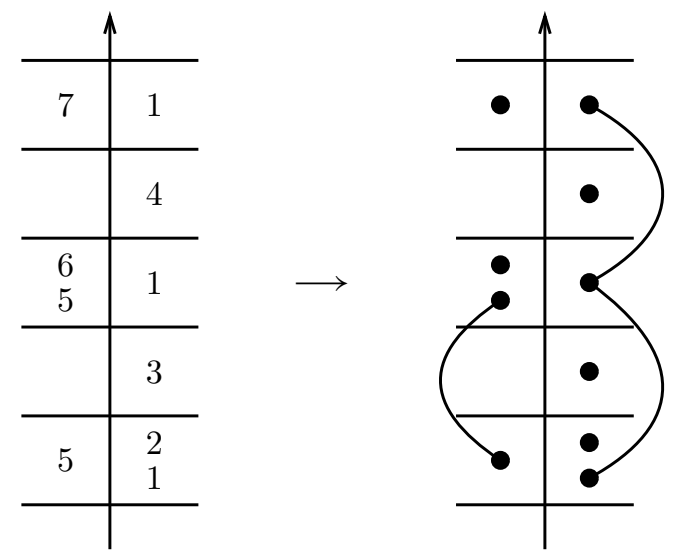

We will consider henceforth (numerical) entries and beads (thus connected by arcs) as equivalent, since both representations carry the same information.

Then there are no overcrossing arcs (again by the Jordan curve theorem, since every arc can be thought of as lying within the same region):

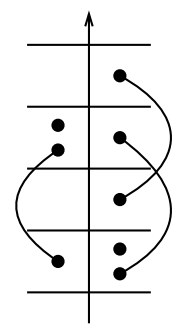

Algebraic 83 Geometric Topology, Volume 6 (2006) 
Finally, no two beads in the same row and side are equivalent (because $R$ is not in a pair of separating regions in $D^{\prime}$ ):

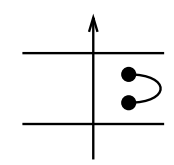

On one of the sides the equivalence arcs may look (after rotating by $90^{\circ}$ ) like

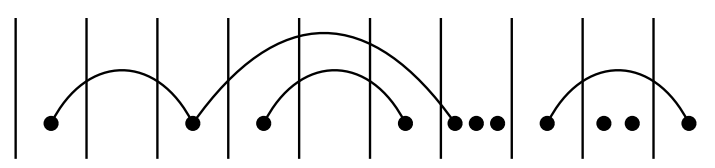

Assume now two consecutive entries on one side are equivalent. The above restrictions imply that

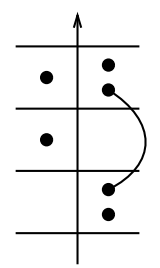

the first one is the last of some row (on some side), and the next one is the first of the second next row (on the same side), with the row in between being empty (on the same side).

If in particular three consecutive entries are equivalent, then the middle one is single on its side and row.

Consider the following transformation on the equivalence arcs (for a moment disregarding the row structure) on both sides.

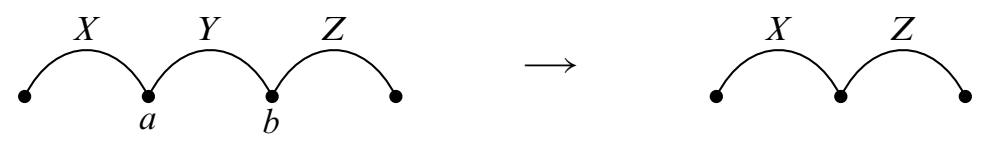

This transformation eliminates a middle one in a series of 4 consecutive equivalent entries (that is, below the arcs the space is assumed to be empty). We will call an arc like the middle arc $Y$ on the left side and the beads $a, b$ it connects shrinkable. Arcs and beads not representable in this form are called non-shrinkable. We will apply (15), but before this let us count how many times we can do so.

Sublemma 3.10 At most $3 / 8$ of the beads in the table (counted altogether on both sides of the table) are removable by the transformation (15).

Algebraic ${ }^{6} \mathcal{G}$ Geometric Topology, Volume 6 (2006) 
(Note that there are more shrinkable beads than removable ones, as on the right of (15) a shrinkable bead remains.)

Proof Consider a shrinkable arc $Y$ in (15). Such an arc identifies entries $a$ and $b$ two rows apart, so that $a$ and $b$ are single on their row and side, and the row between them is empty (on that side).

Let $A, B$ be the slots of the table in the rows of $a$ and $b$, respectively, but on the opposite side to $a$ and $b$. Let $C$ be the slot in between $A$ and $B$.

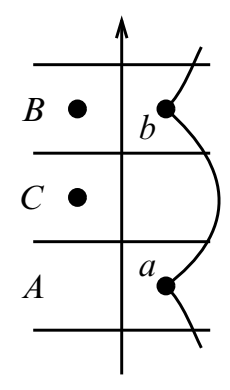

Then by the restrictions there are two alternatives:

(a) $C$ contains at least two entries or

(b) $C$ contains one entry, and at least one of $A$ and $B$ contains at least one entry.

We would like to count how many entries/beads in $A, B, C$ for all shrinkable arcs in (15) we find this way, such that the beads themselves are non-shrinkable. To ensure the correct counting, we must take for each occurrence of (15) each such bead in $A, B$ or $C$ with weight $1 / k$, where $k$ is the number of different fragments (15) for which the bead occurs in (16). Our aim is to group non-shrinkable beads and shrinkable arcs so that the weighted bead count is at least $5 / 3$ per shrinkable arc.

It is easy to see that in both of the above cases any of the entries in $A, B$ or $C$ are non-shrinkable, and that the entries on $C$ are counted once, while those on $A$ or $B$ are counted (once or) at most twice for different fragments (16). This means that the contribution of (15) to the above weighted count of $A, B, C$ beads is at least two, unless only one bead is on $A$ or $B$, and this bead is counted twice (ie $k=2$ ). 
Assume thus the only entry on $A$ or $B$ is counted twice. We have then the following table fragment:

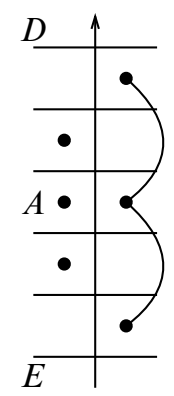

Since $a$ and $b$ are shrinkable, there must be one further row on top (and bottom), whose slot on the opposite side to $a$ and $b$ we call $D$ (and $E$ ), and $D$ (resp. $E$ ) must contain $\geq 2$ beads. (The slot in the row of $D, E$ on the side of $a$ and $b$ is empty because there is at least a terminal arc as $X, Z$ in (15).) These elements may be counted for another shrinkable arc, or not. If there is such an arc, it is on the same side as $a$ and $b$, and part of a longer sequence of consecutive shrinkable arcs (ie $X$ or $Z$ in (15) is also shrinkable).

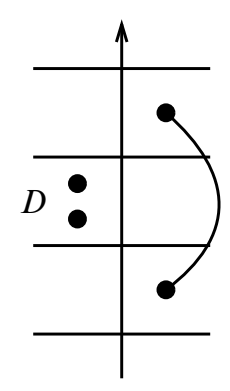

We have thus two or three shrinkable arcs, and have found to them at least 5 entries. It is easy to see that all of these entries are non-shrinkable (because shrinkable arcs connect only single entries on their row, two rows apart, with either neighboring rows being empty on their side of the table). Also any of these entries is not counted again for another shrinkable arc in the above grouping, unless we have:

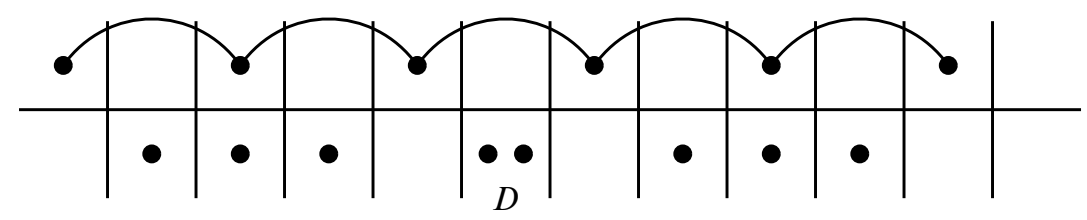

Algebraic $8 \mathcal{G}$ Geometric Topology, Volume 6 (2006) 
(The slot of $D$ may have more than 2 beads.) Then we are in the situation of (17), just with 3 more shrinkable arcs and $\geq 5$ more non-shrinkable beads. We could then argue inductively by the number of consecutive shrinkable arcs.

In summary we found either for a shrinkable arc two non-shrinkable entries (counted with correct weight), or to 2 or 3 shrinkable arcs, 5 non-shrinkable entries (all with weight 1). Thus if there are $l$ arcs/entries in the table removable by (15), at least $5 / 3 l$ other entries are not removable.

Now discard distinction between the left and right side, and the subdivision into rows, and consider the pictures for each side of the table separately. In the remaining picture after the transformation (15) we have no 4 consecutive equivalent beads on the same side.

Consider the equivalence class of the first element. Let it have $t$ elements.

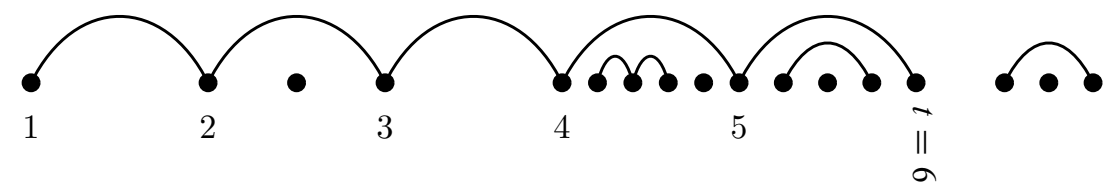

Removing this class, and cutting along the $t$ points, we have $t$ pieces satisfying the following conditions:

- no portion contains 4 consecutive equivalent elements,

- from the first $t-1$ portions, at least every third is non-empty.

Let $n r(n)$ be the minimal number of equivalence classes of $n$ elements under this equivalence relation. We claim

Sublemma 3.11

$$
n r(k) \geq\left\{\begin{array}{cc}
\frac{k}{6}+\frac{1}{2} & k>0 \\
0 & k=0
\end{array}\right\}
$$

Proof We proceed by induction on $k$. We have $n r(0)=0$ and $n r(1)=n r(2)=$ $n r(3)=1$. If $k>3$, we find from the above picture (18) the recursion

$$
\begin{aligned}
& n r(k) \geq 1+\min \left\{\begin{array}{cc}
t & t>0, l_{1}+\cdots+l_{t}=k-t, l_{i} \geq 0, \\
\sum_{i=1} n r\left(l_{i}\right): & \text { at least }\left\lfloor\frac{t-1}{3}\right\rfloor \text { of the } l_{i}>0
\end{array}\right\} \\
& \geq 1+\frac{k-t}{6}+\left\lfloor\frac{t-1}{3}\right\rfloor \cdot \frac{1}{2} \geq 1+\frac{k-t}{6}+\left(\frac{t}{3}-1\right) \cdot \frac{1}{2}=\frac{k}{6}+\frac{1}{2} \text {. }
\end{aligned}
$$

Algebraic $8 \mathcal{G}$ Geometric Topology, Volume 6 (2006) 
We have $r:=$ len $\gamma^{\prime}-1$ rows in the table. As remarked, any third row in the table has at least two entries. Thus the total number of entries is at least

$$
\frac{4 r-2}{3}=\frac{4\left(\operatorname{len} \gamma^{\prime}-1\right)-2}{3}=\frac{4}{3} \operatorname{len} \gamma^{\prime}-2 \text {. }
$$

At most $3 / 8$ of these entries were discarded under the identification (15). By applying the last sublemma, we see that from the remaining at least $5 / 8\left(\frac{4}{3} \operatorname{len} \gamma^{\prime}-2\right)$ entries, at least $1 / 6$ plus one more remained after identifying regions (note that none of the sides of the table was empty, even after (15)).

Thus there are at least

$$
\frac{1}{6} \cdot \frac{5}{8}\left(\frac{4}{3} \operatorname{len} \gamma^{\prime}-2\right)+1=\frac{5}{48}\left(\frac{4}{3} \operatorname{len} \gamma^{\prime}-2\right)+1
$$

other regions not passed by $\gamma^{\prime}$, which have neighbors passed intermediately by $\gamma^{\prime}$. We had in $D^{\prime}$ two more regions, $D_{1,2}^{\prime}$, not passed by $\gamma^{\prime}$ with no neighbored region passed intermediately by $\gamma^{\prime}$. Thus there are at least

$$
\left\lceil\frac{5}{48}\left(\frac{4}{3} \operatorname{len} \gamma^{\prime}-2\right)+3\right\rceil=\left\lceil\frac{5}{36} \operatorname{len} \gamma^{\prime}+\frac{67}{24}\right\rceil \geq \frac{5 \operatorname{len} \gamma^{\prime}+101}{36}
$$

regions not passed by $\gamma^{\prime}$. Then $\gamma^{\prime}$ passes len $\gamma^{\prime}+1$ other regions, so

$$
r\left(D^{\prime}\right) \geq \frac{41}{36}\left(\text { len } \gamma^{\prime}+1\right)+\frac{8}{3},
$$

which leads to the first maximum alternative of the inequality we claimed in (13). The Curve length lemma is now proved.

\section{Genus decreasing bound}

Once we have a bound on the number of regions a curve $\gamma$ of the above specified type passes, we use this bound to obtain a bound on the decrease of genus of a positive knot diagram we can achieve by replacing $\gamma$ by $\gamma_{1}$. In addition to the wave moves, we need the move:

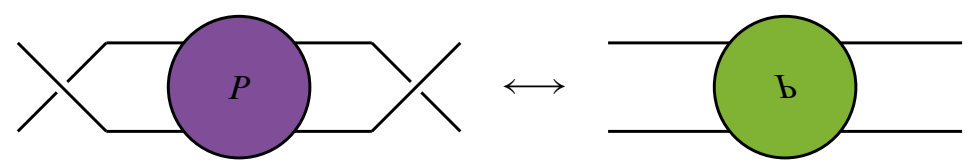

Since for the empty tangle $P$ this is a clasp resolution, we call (19) a generalized clasp reduction move. We can consider a clasp resolution as a special case, included in (19). 
We assume that if $P$ is empty, and the r.h.s. of (19) has nugatory crossings, that we remove all these crossings (ie reduce the diagram).

Theorem 4.1 Let $D$ be a positive diagram of a knot $K$ with $g=g(D)=g(K)>1$.

Then by applying switches of crossings in $D$, and subsequently a wave move (shrinking a bridge/tunnel to length 1), or a move (19), we can obtain a positive diagram $D^{\prime}$ of a positive knot $K^{\prime}$ such that either $g\left(K^{\prime}\right)=g(K)$ and $c\left(D^{\prime}\right)<c(D)$, or $g\left(K^{\prime}\right)<g(K)$ and

$$
g\left(K^{\prime}\right) \geq \frac{6}{13}+\frac{41}{221} g(K)
$$

For the proof we need to quote one more previous result, about the maximal crossing number $c_{g}$ of generators (see Definition 2.12). In [38], a rather rough estimate on the number $d_{g}$ was given, which was later improved in [43] to $d_{g} \leq 6 g-3$. Then in [44] we showed that this inequality is sharp. Since $c_{g} \leq 2 d_{g}$, we have $c_{g} \leq 12 g-6$. Later, in [44], we showed by explicit examples that $c_{g} \geq 10 g-7$, and remarked that at least $c(D) \leq 10 g-6$ if $D$ is a special alternating generator. The work was completed in [36], where, using Hirasawa's algorithm, the maximal generator crossing number was determined (also for links). There the value $10 \mathrm{~g}-7$ was found exact, also for arbitrary generators.

Theorem 4.2 [36] Assume $g \geq 2$. Then $c_{g}=10 g-7$. Moreover, generators of genus $g$ with the maximal number of crossings are always special alternating.

Proof of Theorem 4.1 If $D$ has a bigon region (clasp), we are easily done, since $g\left(K^{\prime}\right) \geq g(K)-1$. So assume $D$ has no such region.

It is easy to see that it suffices also to prove the theorem in case $D$ is prime; the composite case follows easily. For connected sum factors of genus 1 one can use the description in [38]; since all diagrams have a clasp, the genus of $D$ would decrease at most by one.

Choose a minimal admissible curve $\gamma$ in $D$ of length (number of edges in $D \backslash \gamma$ it intersects) len $\gamma>1$, such that start and end region are neighbored (in $D \backslash \gamma$ ). In the proof of the Curve length lemma we argued that an admissible curve always exists. Among these curves $\gamma$ take one of minimal length. Then this curve is one of the type considered in that lemma.

So now we can apply the Curve length lemma. Assume first the first alternative in the maximum of (13) holds. Since $\gamma$ passes len $\gamma+1$ regions (including first and last), 
we can write the estimate as

$$
\text { len } \gamma \leq \frac{36 r\left(D^{\prime}\right)-137}{41}
$$

where $r\left(D^{\prime}\right)$ is the number of regions of $D^{\prime}$, and $D^{\prime}$ is the diagram obtained by repacing $\gamma$ in $D$ by a one-crossing curve $\gamma_{1}$.

We have

$$
r\left(D^{\prime}\right)=2+c\left(D^{\prime}\right)=2+c(D)-\operatorname{len} \gamma+1=3+c(D)-\operatorname{len} \gamma .
$$

Thus

$$
\text { len } \gamma \leq \frac{36(3+c(D)-\operatorname{len} \gamma)-137}{41}=\frac{36(c(D)-\operatorname{len} \gamma)-29}{41}
$$

and

$$
\text { len } \gamma \leq \frac{36 c(D)-29}{77}
$$

We can achieve $D^{\prime}$ to be positive by properly switching crossings of $\gamma$ to become under- or over-crossings, dependingly on how the one crossing of $\gamma_{1}$ is to be switched to be positive. Since $\gamma \cup \gamma_{1}$ gives a closed curve, which can be perturbed to be transversal to $D$ without altering the edges $\gamma$ and $\gamma_{1}$ intersect, a linking number argument shows that the number of crossings on $\gamma$ to be switched is

$$
\frac{\text { len } \gamma}{2} \text { or } \frac{\text { len } \gamma \pm 1}{2} \text {. }
$$

Assume the largest possible value, since we want to have only an upper bound on the number of such crossings. Thus, by switching at most $\frac{\text { len } \gamma+1}{2}$ crossings in $D$, we can simplify it to a positive diagram $D^{\prime}$.

We would like to show now that $D^{\prime}$ is the diagram whose existence was asserted in the theorem.

First, clearly $c\left(D^{\prime}\right)<c(D)$. To see $g\left(D^{\prime}\right) \leq g(D)$, use that $g(D)=g(K)$ and $g\left(D^{\prime}\right)=g\left(K^{\prime}\right)$. Then consider the diagram $D^{\prime \prime}$ obtained from $D$ after the crossing switches on $\gamma^{\prime}$, but before the wave move taking it into $D^{\prime}$ (replacing $\gamma$ by $\gamma_{1}$ ). This is certainly a diagram of $K^{\prime}$, so that $g\left(K^{\prime}\right) \leq g\left(D^{\prime \prime}\right)=g(D)$.

On the other hand, by applying Bennequin's inequality (6) on the diagram $D^{\prime \prime}$ of $K^{\prime}$, we have

$$
g\left(K^{\prime}\right)=g\left(D^{\prime}\right) \geq g(D)-\frac{\operatorname{len} \gamma+1}{2} .
$$


Then, applying (21), we obtain

$$
g\left(D^{\prime}\right) \geq g(D)-\frac{18 c(D)}{77}-\frac{24}{77} .
$$

There is now the possibility to consider the other alternative of the maximum in (13) holds. In this case len $\gamma \leq 3$, so we can switch ( 1 or) 2 crossings, and have a transformation into a diagram $D^{\prime}$ with $c\left(D^{\prime}\right)<c(D)$. By the same argument using (6) as above, $g(D) \geq g\left(D^{\prime}\right) \geq g(D)-2$. The second inequality fits into (22), except if $c(D) \leq 7$. But such $D$ always has a clasp (since it is a knot diagram; by direct verification), which we excluded. So we can ignore the second maximum alternative in (13), and use (22).

The next step is to apply generator estimates. As a preparation, we need to establish two properties of $D^{\prime}$.

Sublemma 4.3 $D^{\prime}$ has no nugatory crossing.

Proof Assume that $c$ were such a crossing.

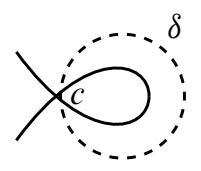

Then there is a nugatory curve $\delta$. Lemma 3.7 implies that $\gamma^{\prime}$ cannot pass twice the region $X$ of $D^{\prime}$ that contains $\delta$ (as in the right picture below). So $\gamma^{\prime}$ remains (as in the left picture) within $X$, and can be isotoped off $\delta$ (within $X$ ). Then $c$ becomes nugatory in $D$, too, a contradiction to its assumed primeness.

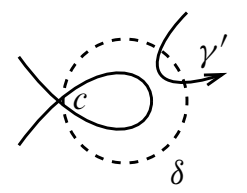

Or

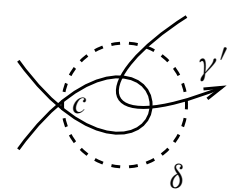

Sublemma 4.4 $D^{\prime}$ admits at most one reducing $\vec{t}_{2}$ move.

Proof Assume there are $3 \sim$-equivalent crossings in $D^{\prime}$. Consider first the case that the crossing of $\gamma_{1}$ is not among them. Let $A$ and $B$ be the (common) non-Seifert circle regions at these crossings. Let $\alpha, \beta, \delta$ be the curves connecting some fixed point in $A$ with some fixed point in $B$ via one of these crossings. Then the join of any 
two of these curves gives a closed curve intersecting $D^{\prime}$ transversely in 2 crossings:

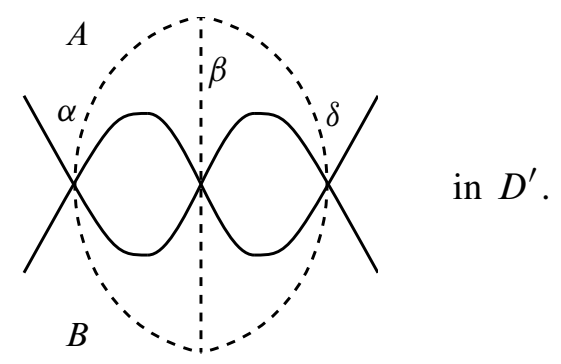

Now we delete $\gamma_{1}$, which does not affect the three crossings and curves, and try to reinstall $\gamma$ so as to obtain $D$.

Since $\gamma^{\prime}$ passes every region of $D^{\prime}$ at most once, if $\gamma^{\prime}$ passes through $\alpha \cup \beta$ without being homotopable off it,

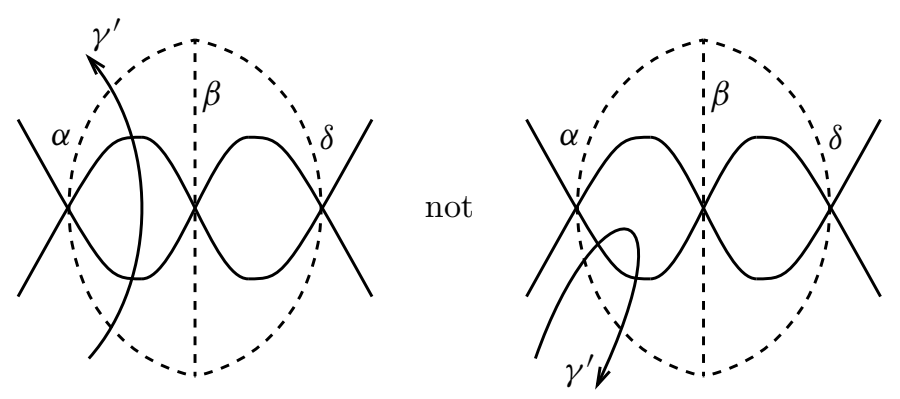

then it cannot pass through $\beta \cup \delta$. Thus up to flype, we have in $D$ a clasp, and can proceed by one crossing change and (19). What appears as a clasp in the above picture may in fact be a non-trivial tangle. Thus there is also the option that $\gamma^{\prime}$ starts and ends within one of these parts:

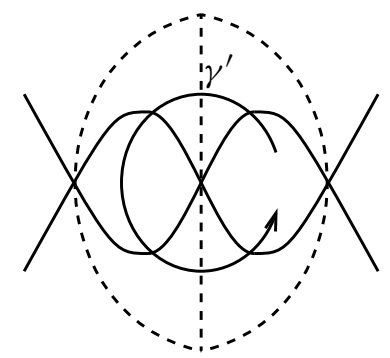

In this case it is $\alpha \cup \delta$ which is not affected (up to homotopy), and then as above (19) applies. 
However, in the second case when one of the three crossings is the one on $\gamma_{1}$, one can have a curve $\gamma^{\prime}$ so that $\gamma$ makes in $D$ all the $\sim$-equivalent crossings inequivalent:

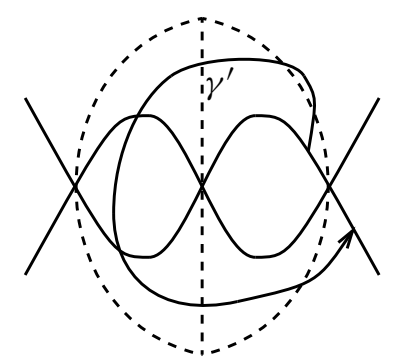

Since there is only one crossing on $\gamma_{1}$, the situation in (23) can happen in at most one $\sim$-equivalence class of $D^{\prime}$, and if there are 4 or more crossings in that class, again (19) can be used.

Now use our previous work on $c_{g}$, the maximal number of crossings of a genus $g$ reduced $\vec{t}_{2}$ irreducible knot diagram (generator) of genus $g$. We have from Theorem 4.2 that $c_{g} \leq 10 g-7$. Therefore, with the previous two sublemmas, we have $c\left(D^{\prime}\right) \leq$ $\log \left(D^{\prime}\right)-5$, and

$$
\begin{aligned}
g\left(D^{\prime}\right) & \geq \frac{c\left(D^{\prime}\right)+5}{10}=\frac{c(D)-\text { len } \gamma+6}{10} \\
& \stackrel{(21)}{\geq} \frac{c(D)-\frac{36 c(D)-29}{77}+6}{10}=\frac{41}{770} c(D)+\frac{491}{770} .
\end{aligned}
$$

This, together with (22), gives

$$
g\left(D^{\prime}\right) \geq \max \left\{g(D)-\frac{18 c(D)+24}{77}, \frac{41}{770} c(D)+\frac{491}{770}\right\} .
$$

To determine the worst possible value of this estimate for fixed $g(D)$, one has to equate both alternatives:

$$
\begin{aligned}
& g(D)=\frac{18 c(D)+24}{77}+\frac{41}{770} c(D)+\frac{491}{770}=\frac{221}{770} c(D)+\frac{731}{770}, \quad \text { and so } \\
& c(D)=\frac{770}{221} g(D)-\frac{731}{221} .
\end{aligned}
$$

Then the r.h.s. of (24) evaluates to

$$
g\left(D^{\prime}\right) \geq \frac{41}{770} c(D)+\frac{491}{770}=\frac{41}{770}\left(\frac{770}{221} g(D)-\frac{731}{221}\right)+\frac{491}{770}=\frac{6}{13}+\frac{41}{221} g(D),
$$

which gives the asserted estimate (20). 


\section{Applications}

\subsection{Proof and extension of Theorem 1.3}

We are now done with most of the work concerning Theorem 1.3. Define for $n>1$ the numbers $g_{n}$ by

$$
g_{n}=\left\lfloor\frac{221 n-323}{41}\right\rfloor,
$$

which we will use throughout this section. Note that $n \leq g_{n}$ when $n \geq 2$. As a consequence of Theorem 4.1, we obtain:

Proposition 5.1 For $n>1$, let $X_{n}$ be the set of positive generating diagrams of genus $n, \ldots, g_{n}$. Let $D$ be a positive knot diagram of genus at least $n$. Then there is a sequence of diagrams $D_{0}, D_{0}^{\prime}, D_{1}, D_{1}^{\prime}, \ldots, D_{k-1}^{\prime}, D_{k}$ with $D_{0}=D$ and $D_{k} \in X_{n}$, satisfying the following properties:

(a) $D_{i}$ is positive and $D_{i}^{\prime}$ differs from $D_{i}$ by crossing changes (ie, $D_{i}$ and $D_{i}^{\prime}$ have the same shadow),

(b) $D_{i+1}$ is obtained from $D_{i}^{\prime}$ either by a move (19), or by rerouting a bridge/tunnel to length 1 .

Proof Let $D$ be a positive diagram of genus $\geq n$. If $g(D) \leq g_{n}$, then we need to reduce $D$ only to the generating diagram in whose series it lies, and for this the move (19) (after the proper crossing changes) is enough. So assume $g(D)>g_{n}$. Since $g(D) \in \mathbb{Z}$, we have then

$$
g(D)>\frac{221 n-323}{41}=\frac{221(n-1)-102}{41} .
$$

Then by Theorem 4.1 we can switch crossings in $D=D_{0}$ to a diagram $D_{0}^{\prime}$, which we can transform into a positive diagram $D_{1}$, such that $c\left(D_{1}\right)<c(D), g\left(D_{1}\right) \leq g(D)$, and

$$
g\left(D_{1}\right) \geq \frac{6}{13}+\frac{41}{221} g(D)>n-1 .
$$

So $g\left(D_{1}\right) \geq n$, and we can argue with $D_{1}$ by induction on $g(D)$, and for fixed $g(D)$, inductively over $c(D)$.

Corollary 5.2 For $n>1$ and $g_{n}$ as in (25) we have

$$
\begin{aligned}
\min \{\sigma(K): K \text { positive, } n \leq & \left.g(K) \leq g_{n}\right\}= \\
& \min \{\sigma(K): K \text { positive, } n \leq g(K)\} .
\end{aligned}
$$

Algebraic 83 Geometric Topology, Volume 6 (2006) 
In particular, assume that there is no positive knot of signature $\leq \sigma$ and genus $n, \ldots, g_{n}$. Then there is no positive knot of signature $\sigma$ and genus $\geq n$ at all.

Proof This follows straightforwardly from Proposition 5.1 and the property (7) of $\sigma$.

Proof of Theorem 1.3 Again this is an immediate consequence of Proposition 5.1, as in Corollary 5.2: let $C_{n}$ be the set of knots with positive generating diagrams of genus $n$ to $g_{n}$.

Remark 5.3 The estimates may be improvable by using more consequently the integrality of $c(D)$ and $g(D)$, which is not always guaranteed in the above calculations. This improvement, however, will affect only the absolute term, and expectedly not in a significant way, so that we preferred to largely waive on incorporating this additional effort into our (anyway technical enough) proofs.

More generally, we have:

\section{Theorem 5.4}

(1) Let $v$ be an invariant with the following property: if $D$ is a positive diagram and $D^{\prime}$ is obtained by (some non-zero number of) crossing switches from it, then $v\left(D^{\prime}\right)<v(D)$. Then $v$ has an increasing lower bound on positive knots in terms of the genus of the knot.

(2) If $v\left(D^{\prime}\right) \leq v(D)$, then one can algorithmically partially decide whether any given value of $v$ is attained on positive knots of only finitely many genera.

Examples of invariants satisfying $v\left(D^{\prime}\right)<v(D)$ are the properly scaled Vassiliev invariants $v_{2}$ and $v_{3}$ of degree 2 and 3. In fact, we showed in [41] independently that for either of $v_{2}$ and $v_{3}$ both the premise and conclusion of part (1) of the above theorem hold. (For $v_{2}$ we must exclude $D^{\prime}$ being the mirror image of $D$, but the argument for Theorem 5.4 clearly still works under this small restriction.) It is interesting to reveal that there is in fact a certain causality between both results, which is quite non-evident from the approach in [41].

Note also that the recent signature-type concordance invariants of Ozsváth and Szabó [27] and Rasmussen [28] satisfy $v\left(D^{\prime}\right) \leq v(D)$, though the conclusion we obtain is known. More interestingly, one can apply part (2) of the theorem to the Tristram-Levine signatures, thereby extending our treatment of $\sigma$. 


\subsection{Partial orders of knots}

To conclude with, we mention a relation to the partial orders of Taniyama and CochranGompf. In [45], Taniyama defined a partial order of knots by $K_{1} \succeq K_{2}$ if the set of shadows of diagrams of $K_{1}$ is a (not necessarily proper) superset of the set of shadows of diagrams of $K_{2}$.

The standard fact that any diagram is unknottable by crossing changes means that the unknot is the maximal element in this partial order. Taniyama showed that the trefoil dominates any other knot, and that $5_{1}$ dominates any knot except connected sums of $(p, q, r)$-pretzel knots, $p, q, r$ odd. The first result gives an easy proof that positive knots have positive signature, and the second result amplifies this statement by showing that $\sigma \geq 4$ if $g \geq 2$. What our arguments show can be thought of as a generalization of Taniyama's two results.

Definition 5.5 Call a set $\mathcal{K}_{1}$ of knots dominating a set $\mathcal{K}_{2}$, in notation $\mathcal{K}_{1} \succeq \mathcal{K}_{2}$, if the set of shadows of all diagrams of knots in $\mathcal{K}_{1}$ is a (not necessarily proper) superset of the set of shadows of diagrams of all knots in $\mathcal{K}_{2}$. A set $\mathcal{K}$ of knots is finitely dominated, if there is a finite subset $\mathcal{K}^{\prime} \subset \mathcal{K}$ dominating $\mathcal{K}$.

Theorem 5.6 For all $n \geq 1$, the set $G_{n}$ of knots of canonical genus $\geq n$ is finitely dominated. There is a finite subset $C_{n} \subset G_{n}$ of positive knots, such that $C_{n} \succeq G_{n}$.

Remark 5.7 It is clear that for two knots $K_{1} \succeq K_{2}$ implies $c\left(K_{1}\right) \leq c\left(K_{2}\right)$. Thus any chain of ' $\succeq$ ' has a maximal element. This means also that the subset of maximal elements is a dominating subset. It is not clear, though, that conversely a dominating subset must contain (all, or even any) maximal elements. Definition 5.5 does not imply that for each $K \in \mathcal{K}_{2}$ there is a $K^{\prime} \in \mathcal{K}_{1}$ with $K^{\prime} \succeq K$, because the $K^{\prime}$ we find for different diagram( shadow)s of $K$ may not be the same. Therefore, we do not know if the set $G_{n}$ in Theorem 5.6 has only finitely many maximal elements. (See, however, in contrast Theorem 5.9.)

Proof of Theorem 5.6 Consider $n \geq 3$, since $C_{1}=\left\{3_{1}\right\}$ and $C_{2}=\left\{5_{1}, 3_{1} \# 3_{1}\right\}$ are Taniyama's results.

With $g_{n}$ defined as in (25), let the set $C_{n}$ consist of the positive knots with positive generating diagrams of genus $n, \ldots, g_{n}$, and write $X_{n}$ for the set of these diagrams. Then apply an induction argument (similar to the one in [42, remark 6.2]).

By Proposition 5.1, for any diagram $D$ of genus $\geq n$, there is a sequence of diagrams $D_{0}, \ldots, D_{k}=D$ (we reindexed the subscripts here) such that $D_{0} \in X_{n}$, and $D_{i}$ and 
$D_{i+1}$ differ by crossing changes, followed either by a generalized clasp resolution (19) or a wave-move of a bridge/tunnel to one of length 1 . The problem is how to switch crossings in $D=D_{k}$, so that one can perform all these moves without switching later crossings in between the moves.

To see how to do this, we use induction on $k$. The case $k=0$ is clear. If $k>0$, we know by induction that we can switch crossings in $D_{k-1}$ so that it reduces to $D_{0}$. The case that $D_{k-1}$ is obtained from $D_{k}=D$ by the move (19), it is clear how to choose the crossing switch of $D_{k}$. (Note that the crossings in the tangle $P$ in (19) are flipped around.)

So consider the case that $D_{k-1}=D^{\prime}$ is obtained from $D_{k}=D$ by shrinking a bridge/tunnel. Then we have an $\operatorname{arc} C$ in $D$ which can be shrunk by a wave-move to an $\operatorname{arc} C^{\prime}$ in $D^{\prime}$ of a single crossing $p$. Since one can crossing-switch $C$ prior to the wave-move to pass above or below the rest of the diagram, one can adjust the sign of the crossing $p$ it collapses to. This crossing $p$ in $D^{\prime}=D_{k-1}$ will be switched at most once in the simplification from $D_{k-1}$ to $D_{0}$. If it is switched (resp. not switched) so that the strand of $C^{\prime}$ becomes (resp. remains) an over/undercrossing in $D^{\prime}$, then switch $C$ so that it becomes a bridge/tunnel in $D$.

Remark 5.8 It is clear that one can choose the set $C_{n}$ to consist of alternating knots, instead of positive ones.

A different, but related partial order of knots was introduced by Cochran and Gompf [6]. In their sense, $K_{1} \geq K_{2}$ if $K_{1}$ is concordant to $K_{2}$ inside a 4-manifold with positive intersection form. This occurs for example if $K_{2}$ is obtained from $K_{1}$ by changing a positive crossing to a negative one. The referee pointed us to make a comment in that context, which we finish with. Clearly, as for Taniyama's partial order, our work directly connects also to the one of Cochran-Gompf. From Proposition 5.1 we obtain immediately:

Theorem 5.9 For each $n \geq 1$, the set of positive knots of genus at least $n$ has a finite number of minimal elements in Cochran-Gompf's partial order.

The case $n=1$ is not formally included in the proposition, but is known. Cochran and Gompf showed that a non-trivial positive knot dominates the right-handed trefoil $! 3_{1}$, which is also a simple implication of Taniyama's related theorem. His second theorem similarly shows that any positive knot of genus $>1$ dominates in ' $\geq$ ' one of $! 5_{1}$ or $! 3_{1} \# ! 3_{1}$. In general it is easy to see that if $K_{1}$ is positive, then $K_{2} \succeq K_{1}$ implies $K_{1} \geq K_{2}$. The converse implication is false even if we assume that $K_{2}$ is 
also positive; $K_{1}=! 5_{1}$ and $K_{2}=! 5_{2}$ give an example. (Slightly fancier pairs of a 12 crossing knot $K_{1}$ and a 13 crossing knot $K_{2}$ show that ' $\geq$ ', even for positive knots, also fails to respect the crossing number - in contrast to ' $\succeq$ '; see Remark 5.7.) Still a more detailed study of the relationship between ' $\geq$ ' and ' $\succeq$ ', which seems not to have been undertaken so far, may be worthwhile.

Acknowledgement This paper emerged from work I carried out over a long period at several places. Some part of this work was written during my stay at the University of Toronto in spring 2002, supported by a grant of Deutsche Forschungsgemeinschaft (DFG). The paper was completed at the Graduate School of Mathematical Sciences, University of Tokyo. Its hospitality, the support by Postdoc grant P04300 of Japan Society for the Promotion of Science (JSPS) and of my host Professor T Kohno is also acknowledged. The author is supported by 21 st Century COE Program.

\section{References}

[1] N A'Campo, Generic immersions of curves, knots, monodromy and Gordian number, Inst. Hautes Études Sci. Publ. Math. (1998) 151-169 (1999) MR1733329

[2] D Bennequin, Entrelacements et équations de Pfaff, from: "Third Schnepfenried geometry conference, Vol. 1 (Schnepfenried, 1982)”, Astérisque 107, Soc. Math. France, Paris (1983) 87-161 MR753131

[3] M Boileau, C Weber, Le problème de J. Milnor sur le nombre gordien des næuds algébriques, Enseign. Math. (2) 30 (1984) 173-222 MR767901

[4] M Brittenham, Bounding canonical genus bounds volume, preprint (1998) Available at http://www.math.unl.edu/ mbritten/personal/pprdescr.html

[5] T D Cochran, Noncommutative knot theory, Algebr. Geom. Topol. 4 (2004) 347-398 MR2077670

[6] T D Cochran, R E Gompf, Applications of Donaldson's theorems to classical knot concordance, homology 3-spheres and property P, Topology 27 (1988) 495-512 MR976591

[7] J H Conway, An enumeration of knots and links, and some of their algebraic properties, from: "Computational Problems in Abstract Algebra (Proc. Conf., Oxford, 1967)", Pergamon, Oxford (1970) 329-358 MR0258014

[8] P R Cromwell, Homogeneous links, J. London Math. Soc. (2) 39 (1989) 535-552 MR1002465

[9] P R Cromwell, Positive braids are visually prime, Proc. London Math. Soc. (3) 67 (1993) 384-424 MR1226607

[10] R Crowell, Genus of alternating link types, Ann. of Math. (2) 69 (1959) 258-275 MR0099665 
Genus generators and the positivity of the signature

[11] R Fintushel, R J Stern, Pseudofree orbifolds, Ann. of Math. (2) 122 (1985) 335-364 MR808222

[12] J Franks, R F Williams, Braids and the Jones polynomial, Trans. Amer. Math. Soc. 303 (1987) 97-108 MR896009

[13] D Gabai, Foliations and genera of links, Topology 23 (1984) 381-394 MR780731

[14] C M Gordon, R A Litherland, K Murasugi, Signatures of covering links, Canad. J. Math. 33 (1981) 381-394 MR617628

[15] M Hirasawa, The flat genus of links, Kobe J. Math. 12 (1995) 155-159 MR1391192

[16] F Hirzebruch, Singularities and exotic spheres, from: "Séminaire Bourbaki, Vol. 10", Soc. Math. France, Paris (1995) Exp. No. 314, 13-32 MR1610436

[17] T Kawamura, Relations among the lowest degree of the Jones polynomial and geometric invariants for a closed positive braid, Comment. Math. Helv. 77 (2002) 125-132 MR1898395

[18] D Kreimer, Knots and Feynman diagrams, Cambridge Lecture Notes in Physics 13, Cambridge University Press (2000) MR1778151

[19] P B Kronheimer, T S Mrowka, The genus of embedded surfaces in the projective plane, Math. Res. Lett. 1 (1994) 797-808 MR1306022

[20] J Levine, Knot cobordism groups in codimension two, Comment. Math. Helv. 44 (1969) 229-244 MR0246314

[21] W W Menasco, M B Thistlethwaite, The Tait flyping conjecture, Bull. Amer. Math. Soc. (N.S.) 25 (1991) 403-412 MR1098346

[22] K Murasugi, On the genus of the alternating knot. I, II, J. Math. Soc. Japan 10 (1958) 94-105, 235-248 MR0099664

[23] K Murasugi, On a certain subgroup of the group of an alternating link, Amer. J. Math. 85 (1963) 544-550 MR0157375

[24] K Murasugi, On a certain numerical invariant of link types, Trans. Amer. Math. Soc. 117 (1965) 387-422 MR0171275

[25] T Nakamura, Positive alternating links are positively alternating, J. Knot Theory Ramifications 9 (2000) 107-112 MR1749503

[26] M Ozawa, Closed incompressible surfaces in the complements of positive knots, Comment. Math. Helv. 77 (2002) 235-243 MR1915040

[27] P Ozsváth, Z Szabó, Knot Floer homology and the four-ball genus, Geom. Topol. 7 (2003) 615-639 MR2026543

[28] J Rasmussen, Khovanov homology and the slice genus arXiv:math.GT/0402131

[29] D Rolfsen, Knots and links, Mathematics Lecture Series 7, Publish or Perish, Berkeley, CA (1976) MR0515288 
[30] L Rudolph, Nontrivial positive braids have positive signature, Topology 21 (1982) 325-327 MR649763

[31] L Rudolph, Quasipositivity as an obstruction to sliceness, Bull. Amer. Math. Soc. (N.S.) 29 (1993) 51-59 MR1193540

[32] L Rudolph, Positive links are strongly quasipositive, from: "Proceedings of the Kirbyfest (Berkeley, CA, 1998)", (J Hass, M Scharlemann, editors), Geom. Topol. Monogr. 2 (1999) 555-562 MR1734423

[33] A Stoimenow, Bennequin's inequality and the positivity of the signature, accepted by Trans. Amer. Math. Soc.

[34] A Stoimenow, Diagram genus, generators and applications, preprint

[35] A Stoimenow, Knots of genus two arXiv:math.GT/0303012

[36] A Stoimenow, On the crossing number of semiadequate links, preprint

[37] A Stoimenow, The signature of 2-almost positive knots, J. Knot Theory Ramifications 9 (2000) 813-845 MR1775388

[38] A Stoimenow, Knots of genus one or on the number of alternating knots of given genus, Proc. Amer. Math. Soc. 129 (2001) 2141-2156 MR1825928

[39] A Stoimenow, On the crossing number of positive knots and braids and braid index criteria of Jones and Morton-Williams-Franks, Trans. Amer. Math. Soc. 354 (2002) 3927-3954 MR1926860

[40] A Stoimenow, The crossing number and maximal bridge length of a knot diagram, Pacific J. Math. 210 (2003) 189-199 MR1989075 With an appendix by Mark Kidwell

[41] A Stoimenow, Positive knots, closed braids and the Jones polynomial, Ann. Sc. Norm. Super. Pisa Cl. Sci. (5) 2 (2003) 237-285 MR2004964

[42] A Stoimenow, Gauß diagram sums on almost positive knots, Compos. Math. 140 (2004) 228-254 MR2004131

[43] A Stoimenow, V Tchernov, A Vdovina, The canonical genus of a classical and virtual knot, Geom. Dedicata 95 (2002) 215-225 MR1950891

[44] A Stoimenow, A Vdovina, Counting alternating knots by genus, Math. Ann. 333 (2005) 1-27 MR2169826

[45] K Taniyama, A partial order of knots, Tokyo J. Math. 12 (1989) 205-229 MR1001742

[46] P Traczyk, Nontrivial negative links have positive signature, Manuscripta Math. 61 (1988) 279-284 MR949818

[47] J M van Buskirk, Positive knots have positive Conway polynomials, from: "Knot theory and manifolds (Vancouver, 1983)", Lecture Notes in Math. 1144, Springer, Berlin (1985) 146-159 MR823288

[48] P Vogel, Representation of links by braids: a new algorithm, Comment. Math. Helv. 65 (1990) 104-113 MR1036132 
[49] R F Williams, Lorenz knots are prime, Ergodic Theory Dynam. Systems 4 (1984) 147-163 MR758900

[50] S Yamada, The minimal number of Seifert circles equals the braid index of a link, Invent. Math. 89 (1987) 347-356 MR894383

[51] Y Yokota, Polynomial invariants of positive links, Topology 31 (1992) 805-811 MR1191382

Research Institute for Mathematical Sciences, Kyoto University

Kyoto 606-8502, Japan

stoimeno@kurims.kyoto-u.ac.jp

http://www.kurims.kyoto-u.ac.jp/ stoimeno/

Received: 24 June 2006 